\title{
TORIC RESIDUES AND MIRROR SYMMETRY
}

\author{
VICTOR V. BATYREV AND EVGENY N. MATEROV \\ To Yuri Ivanovich Manin on his 65-th birthday
}

\begin{abstract}
We develop some ideas of Morrison and Plesser and formulate a precise mathematical conjecture which has close relations to toric mirror symmetry. Our conjecture, we call it Toric Residue Mirror Conjecture, claims that the generating functions of intersection numbers of divisors on a special sequence of simplicial toric varieties are power series expansions of some rational functions obtained as toric residues. We expect that this conjecture holds true for all Gorenstein toric Fano varieties associated with reflexive polytopes and give some evidences for that. The proposed conjecture suggests a simple method for computing Yukawa couplings for toric mirror Calabi-Yau hypersurfaces without solving systems of differential equations. We make several explicit computations for Calabi-Yau hypersurfaces in weighted projective spaces and in products of projective spaces.
\end{abstract}

\section{Contents}

1. Introduction 2

2. Toric residues 4

3. Morrison-Plesser moduli spaces 9

\begin{tabular}{lll}
\hline $4 . \quad$ Toric Residue Mirror Conjecture & 14
\end{tabular}

\begin{tabular}{lll}
\hline $5 . \quad$ Hirzebruch surface $\mathbb{F}_{1}$ & 19
\end{tabular}

6. Toric residue and flop 25

$\begin{array}{lll}7 . & \text { Weighted projective spaces } & 28\end{array}$

\begin{tabular}{lll}
\hline 8. & Product of projective spaces & 32
\end{tabular}

9. Yukawa $(d-1)$-point functions for Calabi-Yau hypersurfaces 35

10. Algorithmic calculation of toric residues 40

11. Mirrors of Calabi-Yau hypersurfaces in $\mathbb{P}^{d_{1}} \times \mathbb{P}^{d_{2}} \quad 43$

References 45

Key words and phrases. residues, toric varieties, intersection numbers, mirror symmetry. 2000 Mathematics Subject Classification. Primary 14M25. 


\section{INTRODUCTION}

The mirror symmetry attracts interest of mathematicians because it allows to identify mathematical objects of a very different nature: generating functions for Gromov-Witten invariants of rational curves on Calabi-Yau manifolds $X$ and power series expansions of special functions on periods of the mirror family of Calabi-Yau manifolds $X^{*}$. Many examples of this identification can be computed explicitly for Calabi-Yau hypersurfaces in toric Fano varieties. The general toric mirror construction [Bat2 suggests a duality between Calabi-Yau varieties with Gorenstein canonical singularities. These singularities in general can not be resolved without changing the canonical class. Therefore, a verification of the toric mirror symmetry in full generality requires orbifold versions of quantum cohomology and GromovWitten invariants for singular varieties (cf. [CR1, CR2]). The Mirror Theorem states that power series obtained by these two very different methods are actually the same. It is rather nontrivial to prove the Mirror Theorem even in the very special case of Calabi-Yau quintic 3-folds G2, G4].

In this paper we propose a more elementary mirror symmetry test which is expected to hold for all families of Calabi-Yau hypersurfaces in Gorenstein toric Fano varieties associated with dual pairs of reflexive polytopes. We formulate this test as a mathematical conjecture and call it Toric Residue Mirror Conjecture. The idea of this conjecture is due to Morrison and Plesser [MP] who have checked it for some toric Calabi-Yau hypersurfaces (including the case of Calabi-Yau quintic 3folds). Unfortunately, Morrison and Plesser didn't formulate their ideas in the form of a precise mathematical conjecture. The most important contribution of Morrison and Plesser $[\mathrm{MP}]$ is the construction of a sequence of toric manifolds $\left\{\mathbb{P}_{\beta}\right\}$ associated with pairs $(\mathbb{P}, \beta)$, where $\mathbb{P}$ is a smooth projective $d$-dimensional toric manifold $\mathbb{P}$ and $\beta$ is an element in the monoid of integral points in the Mori cone $K_{\text {eff }}(\mathbb{P})$ of $\mathbb{P}$. Using ideas of Morrison and Plesser, we define a special cohomology class $\Phi_{\beta} \in H^{2\left(\operatorname{dim} \mathbb{P}_{\beta}-d\right)}\left(\mathbb{P}_{\beta}, \mathbb{Q}\right)$ which plays the role of a "virtual fundamental class" in our mirror symmetry test. The manifold $\mathbb{P}_{\beta}$ and the cohomology class $\Phi_{\beta}$ will be called Morrison-Plesser moduli space and Morrison-Plesser class respectively. For our Toric Residue Mirror Conjecture, we will need a slight generalization of the Morrison-Plesser construction for projective simplicial toric varieties $\mathbb{P}$.

Let us make some remarks concerning the relation of our conjecture to the traditional point of view on the mirror symmetry for Calabi-Yau manifolds (see, e.g., [CK]). First of all, it is important to emphasize that Toric Residue Mirror Conjecture can not be obtained as a consequence of the mirror theorems due to Givental and Lian-Liu-Yau [G4, LLY2, LLY3]. On the other hand, it seems that all known methods for proving Mirror Theorem for toric Calabi-Yau hypersurfaces and complete intersections use some versions of the Morrison-Plesser moduli spaces 
$\mathbb{P}_{\beta}$ as naive toric approximations of the Kontsevich moduli spaces of stable maps G1, G2, G3, G4, G5, LLY1, LLY2, LLY3].

This makes impression that our conjecture could provide a helpful intermediate step for future formulations and verifications of the toric mirror symmetry predictions in case of Calabi-Yau varieties with orbifold singularities. We remark that the Toric Residue Mirror Conjecture also suggests a simple method for computing Yukawa $(d-1)$-point functions for $(d-1)$-dimensional toric Calabi-Yau hypersurfaces without using systems of differential equations.

The main advantage of the Toric Residue Mirror Conjecture is its simplicity. One does not need to know much (e.g., Hodge theory, Gromov-Witten invariants and quantum cohomology) in order to understand its statement. Our conjecture uses only the intersection theory on simplicial toric varieties $\mathbb{P}_{\beta}$ and the notion of toric residues which are standard operations in computational commutative algebra. Unfortunately, we were not able to prove our conjecture in general. In this paper, we check it for some classes of reflexive polytopes using direct computations. We hope that a conceptual proof of the Toric Residue Mirror Conjecture for arbitrary reflexive polytopes would be an important contribution to understanding of the mirror symmetry phenomenon.

The paper is organized as follows. In Section 2, we give a review of toric residues and their properties. In Section 3, we discuss Morrison-Plesser moduli spaces $\mathbb{P}_{\beta}$ associated with lattice points $\beta$ in the Mori cone $K_{\text {eff }}(\mathbb{P})$ of a simplicial toric variety $\mathbb{P}$. In Section 4, we formulate the Toric Residue Mirror Conjecture and give some evidences for it. In Sections 5 and 6, we illustrate our conjecture for some simplest interesting cases. In Sections 7 and 8, we prove the Toric Residue Mirror Conjecture for Calabi-Yau hypersurfaces in weighted projective spaces and in product of projective spaces. Section 9 explains applications of our conjecture to the toric mirror symmetry and to the computations of Yukawa couplings for toric CalabiYau hypersurfaces. Some examples of these computations are given in Sections 10 and 11.

We remark that the Toric Residue Mirror Conjecture can be formulated in the same generality also for toric Calabi-Yau complete intersections associated with nefpartitions of reflexive polytopes. The details of this generalization will be published in the forthcoming paper $[\mathrm{BM}]$.

Acknowledgments. We would like to thank A. Mavlyutov for suggesting us to use his formula for computation of Yukawa couplings and for pointing out us on the correction in constants in this formula. We thank E. Cattani, A. Dickenstein and M. Passare for their remarks and corrections concerning preprint version of this work. The authors were supported by DFG, Forschungsschwerpunkt "Globale 
Methoden in der komplexen Geometrie". E. Materov was partially supported by RFBR Grant 00-15-96140.

\section{TORIC RESIDUES}

The notion of toric residue was introduced by D. Cox using homogeneous coordinates on toric varieties [Cox2]. In this paper, we prefer another approach which uses the affine coordinates $t_{1}, \ldots, t_{d}$ on the algebraic torus $\mathbb{T}=\operatorname{Spec} \mathbb{C}\left[t_{1}^{ \pm 1}, \ldots, t_{d}^{ \pm 1}\right]$.

Denote by $M$ the group of characters of $\mathbb{T}$ which is isomorphic to $\mathbb{Z}^{d}$. Let $\Delta$ be a $d$-dimensional convex polytope in $M_{\mathbb{R}}=M \otimes \mathbb{R}$ with vertices in $M$.

Definition 2.1. We denote by

$$
S_{\Delta}=\bigoplus_{k=0}^{\infty} S_{\Delta}^{k}
$$

the graded subring in $\mathbb{C}\left[t_{0}, t_{1}^{ \pm 1}, \ldots, t_{d}^{ \pm 1}\right]$ whose $k$-th homogeneous component $S_{\Delta}^{k}$ is spanned as $\mathbb{C}$-vector space by all monomials $t_{0}^{k} t_{1}^{m_{1}} \cdots t_{d}^{m_{d}}$ such that the lattice point $\left(m_{1}, \ldots, m_{d}\right)$ is contained in $k \Delta$.

Definition 2.2. The variety $\mathbb{P}_{\Delta}:=\operatorname{Proj} S_{\Delta}$ is called the projective toric variety associated with the polytope $\Delta$. We denote by $\mathcal{O}_{\mathbb{P}_{\Delta}}(1)$ the ample invertible sheaf on $\mathbb{P}_{\Delta}$ corresponding to the graded $S_{\Delta}$-module $S_{\Delta}(-1)$.

Definition 2.3. Denote by $I_{\Delta}=\bigoplus_{k=0}^{\infty} I_{\Delta}^{k}$ the graded ideal in $S_{\Delta}$ whose $k$-th homogeneous component $I_{\Delta}^{k}$ is spanned over $\mathbb{C}$ by all monomials $t_{0}^{k} t_{1}^{m_{1}} \cdots t_{d}^{m_{d}}$ such that $\left(m_{1}, \ldots, m_{d}\right)$ is contained in the interior of $k \Delta$.

It is known that $S_{\Delta}$ is a Cohen-Macaulay ring and $I_{\Delta}$ is its dualizing module Dan. Let $g_{0}(t), g_{1}(t), \ldots, g_{d}(t)$ be generic Laurent polynomials in $\mathbb{C}\left[t_{1}^{ \pm 1}, \ldots, t_{d}^{ \pm 1}\right]$ with supports in $\Delta$ such that

$$
G=\left(G_{0}, G_{1}, \ldots, G_{d}\right):=\left(t_{0} g_{0}(t), t_{0} g_{1}(t), \ldots, t_{0} g_{d}(t)\right) \subset S_{\Delta}^{1}
$$

is a regular sequence in $S_{\Delta}$. We can consider the polynomials $G_{0}, G_{1}, \ldots, G_{d}$ also as global sections of $\mathcal{O}_{\mathbb{P}_{\Delta}}(1)$ on $\mathbb{P}_{\Delta}$ having no common zeros.

Since $G$ is also a regular sequence for the dualizing module $I_{\Delta}$, we obtain two finite-dimensional graded $\mathbb{C}$-vector spaces

$$
S_{G}:=S_{\Delta} /\left\langle G_{0}, G_{1}, \ldots, G_{d}\right\rangle S_{\Delta}, \quad I_{G}:=I_{\Delta} /\left\langle G_{0}, G_{1}, \ldots, G_{d}\right\rangle I_{\Delta},
$$

where $I_{G}$ is a dualizing module of the Artinian graded ring $S_{G}$ (see Bat1, Proposition 9.4]). One has a duality

$$
S_{G}^{k} \times I_{G}^{d+1-k} \rightarrow I_{G}^{d+1}, \quad k=0, \ldots, d,
$$


where $I_{G}^{d+1}$ is a 1 -dimensional $\mathbb{C}$-vector space. The Jacobian

$$
J_{G}:=\operatorname{det}\left(t_{i} \frac{\partial G_{j}}{\partial t_{i}}\right)_{0 \leq i, j \leq d}=t_{0}^{d+1} \operatorname{det}\left(\begin{array}{cccc}
g_{0} & g_{1} & \cdots & g_{d} \\
t_{1} \frac{\partial g_{0}}{\partial t_{1}} & t_{1} \frac{\partial g_{1}}{\partial t_{1}} & \cdots & t_{1} \frac{\partial g_{d}}{\partial t_{1}} \\
\vdots & \vdots & \ddots & \vdots \\
t_{d} \frac{\partial g_{0}}{\partial t_{d}} & t_{d} \frac{\partial g_{1}}{\partial t_{d}} & \cdots & t_{d} \frac{\partial g_{d}}{\partial t_{d}}
\end{array}\right)
$$

is an element of $S_{\Delta}^{d+1}$. One can easily check that $J_{G}$ is contained in $I_{\Delta}^{d+1} \subset S_{\Delta}^{d+1}$ (see [CDS, Proposition 1.2]) and the class of $J_{G}$ in $I_{G}$ spans $I_{G}^{d+1}$.

We define the toric residue in the following algebraic way:

Definition 2.4. Let $\operatorname{Vol}(\Delta)$ be the normalized volume of $\Delta$, i.e., $d ! \cdot($ volume of $\Delta$ ). The $\mathbb{C}$-linear map

$$
\operatorname{Res}_{G}: I_{\Delta}^{d+1} \rightarrow \mathbb{C},
$$

vanishing on the $\mathbb{C}$-subspace $\left\langle G_{0}, G_{1}, \ldots, G_{d}\right\rangle I_{\Delta}^{d} \subset I_{\Delta}^{d+1}$ and sending $J_{G}$ to $\operatorname{Vol}(\Delta)$ is called the toric residue. This map establishes a canonical isomorphism

$$
I_{G}^{d+1}=I_{\Delta}^{d+1} /\left\langle G_{0}, G_{1}, \ldots, G_{d}\right\rangle I_{\Delta}^{d} \cong \mathbb{C} .
$$

Remark 2.5. This algebraic definition of the toric residue works as well for any algebraically closed field $k$ of characteristic 0 . We compare it with the definition given by D. Cox in Section 9 .

There exists a more geometric point of view on the toric residue. For this, one remarks that the coherent sheaf $\widetilde{I_{\Delta}}$ associated with the graded $S_{\Delta}$-module $I_{\Delta}$ is exactly the sheaf $\Omega_{\mathbb{P}_{\Delta}}^{d}$ of Zariski differential $d$-forms (or dualizing sheaf) on $\mathbb{P}_{\Delta}$ Dan. Comparing the pairing

$$
H^{d}\left(\mathbb{P}_{\Delta}, \Omega_{\mathbb{P}_{\Delta}}^{d}\right) \times H^{0}\left(\mathbb{P}_{\Delta}, \mathcal{O}_{\mathbb{P}_{\Delta}}\right) \rightarrow H^{d}\left(\mathbb{P}_{\Delta}, \Omega_{\mathbb{P}_{\Delta}}^{d}\right)
$$

with the pairing

$$
I_{G}^{d+1} \times S_{G}^{0} \rightarrow I_{G}^{d+1}
$$

we can canonically identify the 1 -dimensional $\mathbb{C}$-space $I_{G}^{d+1}$ with $H^{d}\left(\mathbb{P}_{\Delta}, \Omega_{\mathbb{P}_{\Delta}}^{d}\right)$. The regular sequence $G$ defines a finite morphism of degree $\operatorname{Vol}(\Delta)$

$$
\Psi: \mathbb{P}_{\Delta} \rightarrow \mathbb{P}^{d}, \quad p \mapsto\left(G_{0}(p): G_{1}(p): \cdots: G_{d}(p)\right) .
$$

Consider the Čech cocycle

$$
\alpha=\frac{z_{0}^{d}}{z_{1} \cdots z_{d}} d\left(\frac{z_{1}}{z_{0}}\right) \wedge \cdots \wedge d\left(\frac{z_{d}}{z_{0}}\right)
$$


in the standard open covering $\mathcal{U}=\left\{z_{i} \neq 0\right\}_{i=0, \ldots, d}$ of $\mathbb{P}^{d}$ with the homogeneous coordinates $z_{0}, z_{1}, \ldots, z_{d}$. One can show that $\alpha$ determines a generator of $H^{d}\left(\mathbb{P}^{d}, \Omega_{\mathbb{P}^{d}}^{d}\right)$ [Ha1, Chapter III, $\S 7]$. It is easy to check that the map

$$
\Psi^{*}: H^{d}\left(\mathbb{P}^{d}, \Omega_{\mathbb{P}^{d}}^{d}\right) \rightarrow H^{d}\left(\mathbb{P}_{\Delta}, \Omega_{\mathbb{P}_{\Delta}}^{d}\right)
$$

sends $\alpha$ to the Čech cocycle

$$
\Psi^{*}(\alpha)=\frac{J_{G}}{G_{0} \cdots G_{d}} \frac{d t_{1}}{t_{1}} \wedge \cdots \wedge \frac{d t_{d}}{t_{d}}
$$

in the covering $\mathcal{U}^{\prime}=\left\{G_{i} \neq 0\right\}_{i=0, \ldots, d}$ of $\mathbb{P}_{\Delta}$. Since the sheaf $\Omega_{\mathbb{P}_{\Delta}}^{d}$ is dualizing, there exists a canonical trace map $T_{\mathbb{P}_{\Delta}}: H^{d}\left(\mathbb{P}_{\Delta}, \Omega_{\mathbb{P}_{\Delta}}^{d}\right) \rightarrow \mathbb{C}$ (see [Ha1, Chapter III, $\left.\S 7\right]$ ). By the property of the trace map

$$
T_{\mathbb{P}_{\Delta}}\left(\left[\Psi^{*}(\alpha)\right]\right)=\operatorname{deg}(\Psi) T_{\mathbb{P}^{d}}([\alpha])=\operatorname{Vol}(\Delta) T_{\mathbb{P}^{d}}([\alpha])
$$

(see [Ha2, Chapter III]). This naturally explains the normalization $\operatorname{Res}_{G}\left(J_{G}\right)=$ $\operatorname{Vol}(\Delta)$ in Definition 2.4.

Let us review some properties of the toric residue.

Choose a regular sequence $G=\left(G_{0}, G_{1}, \ldots, G_{d}\right)$ in $S_{\Delta}^{1}$ defined by Laurent polynomials $g_{0}(t), g_{1}(t), \ldots, g_{d}(t)$ supported in $\Delta$ as above. It follows from the regularity of $G$ that the set $V_{g}$ of common zeros of $G_{1}, \ldots, G_{d}$ in $\mathbb{P}_{\Delta}$ is finite. Next statement follows immediately from [CCD, Theorem 0.4] and [CDS, Proposition 1.3] and claims that toric residue can be expressed as a sum of local Grothendieck residues.

Theorem 2.6. Let $p\left(t_{1}, \ldots, t_{d}\right)$ be a Laurent polynomial with support in the interior of $(d+1) \Delta$. We set $P:=t_{0}^{d+1} p\left(t_{1}, \ldots, t_{d}\right) \in I_{\Delta}^{d+1}$ and choose a sufficiently generic regular sequence $\left(G_{0}, G_{1}, \ldots, G_{d}\right)=\left(t_{0} g_{0}, t_{0} g_{1}, \ldots, t_{0} g_{d}\right) \subset S_{\Delta}^{1}$ such the set $V_{g}$ of common zeros of $G_{1}, \ldots, G_{d}$ in $\mathbb{P}_{\Delta}$ is contained in $\mathbb{T} \cong\left(\mathbb{C}^{*}\right)^{d}$. Then

$$
\operatorname{Res}_{G}(P)=\sum_{\xi \in V_{g}} \operatorname{res}_{g, \xi}\left(\omega_{P}\right)
$$

where

$$
\omega_{P}=\frac{p / g_{0}}{g_{1} \cdots g_{n}} \frac{d t_{1}}{t_{1}} \wedge \cdots \wedge \frac{d t_{d}}{t_{d}}
$$

and $\operatorname{res}_{g, \xi}\left(\omega_{P}\right)$ is the local Grothendieck residue of the form $\omega_{P}$ at the point $\xi \in V_{g}$.

In particular, if all the common roots of $g_{1}, \ldots, g_{d}$ in $\mathbb{T}$ are simple, then

$$
\operatorname{Res}_{G}(P)=\sum_{\xi \in V_{g}} \frac{p(\xi)}{g_{0}(\xi) J_{g}^{0}(\xi)}
$$

where

$$
J_{g}^{0}:=\operatorname{det}\left(t_{i} \frac{\partial g_{j}}{\partial t_{i}}\right)_{1 \leq i, j \leq d}
$$


Let $A$ be a finite subset in $\Delta \cap M$ which contains all vertices of $\Delta$. Write each of the polynomials $g_{0}(t), g_{1}(t), \ldots, g_{d}(t)$ as

$$
g_{j}(t)=\sum_{m \in A} a_{m}^{(j)} t^{m}, \quad j=0,1, \ldots, d .
$$

Let $\mathbb{Q}[a]$ be the polynomial ring in the variables $a_{m}^{(j)}(m \in A, 0 \leq j \leq d)$. Denote by $\mathcal{R}_{g_{0}, \ldots, g_{d}}(a) \in \mathbb{Q}[a]$ the (unmixed) sparse A-resultant of $g_{0}, \ldots, g_{d}$ defined in GKZ, $\S 8.2]$. The following statement, which is a reformulation of [CDS, Theorem 1.4] and [Di, Proposition 3.5], claims that toric residue is a rational function in coefficients of Laurent polynomials $g_{0}, g_{1}, \ldots, g_{d}$.

Theorem 2.7. For any interior lattice point $m$ in $(d+1) \Delta$, there exists a polynomial $Q_{m}(a) \in \mathbb{Q}[a]$ such that

$$
\operatorname{Res}_{G}\left(t_{0}^{d+1} t^{m}\right)=\frac{Q_{m}(a)}{\mathcal{R}_{g_{0}, \ldots, g_{d}}(a)}
$$

We will be mostly interested in toric residues in the special case when the regular sequence $F=\left(F_{0}, F_{1}, \ldots, F_{d}\right)$ of elements in $S_{\Delta}^{1}$ is constructed as follows. Take a generic Laurent polynomial

$$
f(t)=\sum_{m \in A} a_{m} t^{m} \in \mathbb{C}\left[t_{1}^{ \pm 1}, \ldots, t_{d}^{ \pm 1}\right]
$$

and define

$$
F_{0}:=t_{0} f(t), F_{1}:=t_{0} f_{1}(t), \ldots, F_{d}:=t_{0} f_{d}(t),
$$

where $f_{i}(t):=t_{i} \partial f / \partial t_{i}(1 \leq i \leq d)$. In this case, the Jacobians $J_{F}, J_{F}^{0}$ become Hessians

$$
H_{f}:=\operatorname{det}\left(\left(t_{i} \frac{\partial}{\partial t_{i}}\right)\left(t_{j} \frac{\partial}{\partial t_{j}}\right) t_{0} f\right)_{0 \leq i, j \leq d}, H_{f}^{0}:=\operatorname{det}\left(t_{j} \frac{\partial f_{i}}{\partial t_{j}}\right)_{1 \leq i, j \leq d} .
$$

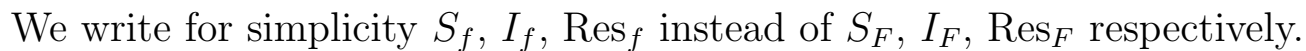

The principal $A$-determinant $E_{A}(f)$ of polynomial $f(t)$ is defined to be the sparse resultant $\mathcal{R}_{f, f_{1}, \ldots, f_{d}}(a)$ of polynomials $f(t), f_{1}(t), \ldots, f_{d}(t)$ GKZ, $\left.\S 10.1\right]$. It follows from [Bat1, §4] that principal $A$-determinant $E_{A}(f)$ is nonzero if and only if $F_{0}, F_{1}, \ldots, F_{d}$ form a regular sequence in $S_{\Delta}^{1}$. In the latter case the polynomial $f(t)$ will be called $\Delta$-regular. Note that $\Delta$-regularity of $f$ guarantees that all critical points of $f$ are isolated.

Remark 2.8. There exists another definition of $\Delta$-regularity of a Laurent polynomial $f=\sum_{m \in A} a_{m} t^{m}$. For any face $\Gamma$ of $\Delta$, we define the Laurent polynomial

$$
f_{\Gamma}:=\sum_{m \in A \cap \Gamma} a_{m} t^{m}
$$


The polynomial $f$ is called $\Delta$-regular if

$$
\left\{t \in \mathbb{T}: f_{\Gamma}(t)=\frac{\partial f_{\Gamma}}{\partial t_{1}}(t)=\cdots=\frac{\partial f_{\Gamma}}{\partial t_{d}}(t)=0\right\}=\emptyset
$$

for all faces $\Gamma \subseteq \Delta$. This definition is equivalent to the previous one, because $E_{A}(f)$ is equal to the product of discriminants [GKZ, Chapter 10, Theorem 1.2]

$$
E_{A}(f)= \pm \prod_{\Gamma \subseteq \Delta} D_{A \cap \Gamma}\left(f_{\Gamma}\right)^{\mu_{\Gamma}}, \quad \mu_{\Gamma} \in \mathbb{Z}_{>0}
$$

and for each face $\Gamma \subseteq \Delta$ the condition (3) holds if and only if the discriminant $D_{A \cap \Gamma}\left(f_{\Gamma}\right)$ is nonzero.

The statements of theorems 2.6 and 2.7 for the toric residue $\operatorname{Res}_{f}$ can be summarized as follows:

Theorem 2.9. Let $P=t_{0}^{d+1} p\left(t_{1}, \ldots, t_{d}\right)$ be an arbitrary element in $I_{\Delta}^{d+1}$. Then

(i) for any sufficiently generic $\Delta$-regular Laurent polynomial $f(t)$ such that the set $V_{f}$ of all common zeros of $F_{1}, \ldots, F_{d}$ in $\mathbb{P}_{\Delta}$ is contained in $\mathbb{T} \cong\left(\mathbb{C}^{*}\right)^{d}$ and any critical point $\xi \in V_{f}$ of $f$ is nondegenerate (i.e., $H_{f}^{0}(\xi) \neq 0$ ), one has

$$
\operatorname{Res}_{f}(P)=\sum_{\xi \in V_{f}} \frac{p(\xi)}{f(\xi) H_{f}^{0}(\xi)} .
$$

(ii) the toric residue $\operatorname{Res}_{f}(P)$ is a rational function in the coefficients of the polynomials $p(t)$ and $f(t)$. In particular, for any interior lattice point $m$ in $(d+1) \Delta$, there exists a polynomial $Q_{m}(a) \in \mathbb{Q}[a]$ such that

$$
\operatorname{Res}_{f}\left(t_{0}^{d+1} t^{m}\right)=\frac{Q_{m}(a)}{E_{A}(f)}
$$

Let $N:=\operatorname{Hom}(M, \mathbb{Z})$ be the dual to $M$ lattice and $N_{\mathbb{R}}:=N \otimes \mathbb{R}$. We denote by $\langle *, *\rangle$ the natural pairing $M_{\mathbb{R}} \times N_{\mathbb{R}} \rightarrow \mathbb{R}$.

Definition 2.10 (Bat2). A polytope $\Delta \subset M_{\mathbb{R}}$ with vertices in $M$ is called reflexive if it contains 0 as interior point and its polar polytope

$$
\Delta^{*}=\left\{y \in N_{\mathbb{R}}:\langle x, y\rangle \geq-1, \quad \forall x \in \Delta\right\} \subset N_{\mathbb{R}}
$$

has vertices in $N$. We remark that $\Delta$ is reflexive if and only if $\mathbb{P}_{\Delta}$ is a Gorenstein toric Fano variety and $\mathcal{O}_{\mathbb{P}_{\Delta}}(1)$ is the anticanonical sheaf on $\mathbb{P}_{\Delta}$.

The reflexivity of $\Delta$ implies that $\Delta^{*}$ is also reflexive and $\left(\Delta^{*}\right)^{*}=\Delta$. We will demand that the finite subset $A \subset M \cap \Delta$ contains not only all the vertices of $\Delta$, but also the lattice point 0 as interior. 
Now consider the toric residue $\operatorname{Res}_{f}$ in the special case when the support polytope $\Delta$ of $f$ is reflexive. In this case, $I_{\Delta}$ is the principal ideal in $S_{\Delta}$ generated by $t_{0}$. This implies that $H_{f} \in I_{\Delta}^{d+1} \subset S_{\Delta}^{d+1}$ can be uniquely written as product $t_{0} H_{f}^{\prime}$, where

$$
H_{f}^{\prime}=t_{0}^{d} \operatorname{det}\left(\begin{array}{cccc}
f & f_{1} & \cdots & f_{d} \\
f_{1} & t_{1} \frac{\partial f_{1}}{\partial t_{1}} & \cdots & t_{1} \frac{\partial f_{d}}{\partial t_{1}} \\
\vdots & \vdots & \ddots & \vdots \\
f_{d} & t_{d} \frac{\partial f_{1}}{\partial t_{d}} & \cdots & t_{d} \frac{\partial f_{d}}{\partial t_{d}}
\end{array}\right) \in S_{\Delta}^{d} .
$$

The ring $S_{\Delta}$ is Gorenstein and the multiplication in Artinian graded ring $S_{f}$ defines the perfect pairings

$$
S_{f}^{k} \times S_{f}^{d-k} \rightarrow S_{f}^{d}, \quad k=0, \ldots, d,
$$

where $S_{f}^{d}$ is a 1-dimensional $\mathbb{C}$-vector space generated by the class of $H_{f}^{\prime}$. By abuse of notations, we denote by $\operatorname{Res}_{f}$ also the $\mathbb{C}$-linear map

$$
\operatorname{Res}_{f}: S_{\Delta}^{d} \rightarrow \mathbb{C}
$$

vanishing on the subspace $\left\langle F_{0}, F_{1}, \ldots, F_{d}\right\rangle S_{\Delta}^{d-1} \subset S_{\Delta}^{d}$ such that $\operatorname{Res}_{f}\left(H_{f}^{\prime}\right)=\operatorname{Vol}(\Delta)$. This map induces a canonical isomorphism

$$
S_{f}^{d}=S_{\Delta}^{d} /\left\langle F_{0}, F_{1}, \ldots, F_{d}\right\rangle S_{\Delta}^{d-1} \cong \mathbb{C}
$$

\section{MORRISOn-Plesser MOdUli SPACES}

Let $\mathbb{P}=\mathbb{P}_{\Sigma}$ be a $d$-dimensional projective simplicial toric variety over $\mathbb{C}$ defined by a simplicial fan $\Sigma$ whose 1-dimensional cones are generated by integral vectors $e_{1}, \ldots, e_{n} \in N$. There exists a canonical embedding $M=\operatorname{Hom}(N, \mathbb{Z}) \hookrightarrow \mathbb{Z}^{n}$ defined by

$$
m \mapsto\left(\left\langle m, e_{1}\right\rangle, \ldots,\left\langle m, e_{n}\right\rangle\right)
$$

which gives rise to the short exact sequence

$$
0 \rightarrow M \rightarrow \mathbb{Z}^{n} \rightarrow \mathrm{Cl}(\mathbb{P}) \rightarrow 0
$$

where $\mathrm{Cl}(\mathbb{P})$ is canonically isomorphic to the group of Weil divisor classes on $\mathbb{P}$ modulo linear equivalence.

Let $G \subset\left(\mathbb{C}^{*}\right)^{n}$ be the diagonalizable algebraic group over $\mathbb{C}$ with the character group $\mathrm{Cl}(\mathbb{P})$. Then $\mathbb{P}$ can be described as a quotient $U(\Sigma) / G$ (space of orbits), where $U(\Sigma)$ is an open dense subset in $\mathbb{C}^{n}$ and the action of $G$ on $U(\Sigma)$ is induced by the embedding $G \hookrightarrow\left(\mathbb{C}^{*}\right)^{n}$ defined by the epimorphism $\mathbb{Z}^{n} \rightarrow \mathrm{Cl}(\mathbb{P})$. The standard affine coordinates $z_{1}, \ldots, z_{n}$ on $\mathbb{C}^{n}$ determine homogeneous coordinates on $\mathbb{P}$ Cox1. Every equation $z_{j}=0(1 \leq j \leq n)$ defines a Weil (QQQ-Cartier) divisor $D_{j}$ on $\mathbb{P}$. We denote by $\chi_{1}, \ldots, \chi_{n}$ the characters of $G$ defining the representation of $G$ in $G L(n, \mathbb{C})$. These characters correspond to the Weil divisor classes 
$\left[D_{1}\right], \ldots,\left[D_{n}\right] \in \mathrm{Cl}(\mathbb{P})$. Let $R(\Sigma)$ be the subgroup in $\mathbb{Z}^{n}$ consisting of all lattice vectors $\lambda=\left(l_{1}, \ldots, l_{n}\right)$ such that $l_{1} e_{1}+\cdots+l_{n} e_{n}=0$ and $N^{\prime}$ the sublattice of finite index in $N$ generated by $e_{1}, \ldots, e_{n}$. We have the exact sequence

$$
0 \rightarrow R(\Sigma) \rightarrow \mathbb{Z}^{n} \rightarrow N \rightarrow N / N^{\prime} \rightarrow 0
$$

where the middle map is $\left(l_{1}, \ldots, l_{n}\right) \mapsto \sum_{j=1}^{n} l_{j} e_{j}$. Tensoring this sequence by $\mathbb{Q}$, one gets

$$
0 \rightarrow R(\Sigma) \otimes \mathbb{Q} \rightarrow \mathbb{Q}^{n} \rightarrow N \otimes \mathbb{Q} \rightarrow 0
$$

Comparing the latter with the exact sequence

$$
0 \rightarrow M \otimes \mathbb{Q} \rightarrow \mathbb{Q}^{n} \rightarrow \mathrm{Cl}(\mathbb{P}) \otimes \mathbb{Q} \rightarrow 0
$$

we obtain the canonical isomorphisms

$$
\operatorname{Hom}(R(\Sigma), \mathbb{Q}) \cong \mathrm{Cl}(\mathbb{P}) \otimes \mathbb{Q} \cong \operatorname{Pic}(\mathbb{P}) \otimes \mathbb{Q} \cong H^{2}(\mathbb{P}, \mathbb{Q}) .
$$

Therefore, $R(\Sigma)_{\mathbb{Q}}:=R(\Sigma) \otimes \mathbb{Q}$ can be identified with the rational homology group $H_{2}(\mathbb{P}, \mathbb{Q})$. For any $\mathbb{Q}$-divisor $D=\sum_{j=1}^{n} c_{j} D_{j}\left(c_{j} \in \mathbb{Q}\right)$ and for any $\lambda=\left(l_{1}, \ldots, l_{n}\right) \in$ $R(\Sigma)_{\mathbb{Q}}$, one has the intersection number

$$
(D, \lambda)=\sum_{i=1}^{n} c_{i} l_{i} \in \mathbb{Q} .
$$

If $H$ is an ample Cartier divisor on $\mathbb{P}$, then the fan $\Sigma$ can be obtained as a normal fan for the simple $d$-dimensional polytope

$$
\Delta_{H}:=\left\{\left(x_{1}, \ldots, x_{n}\right) \in \mathbb{R}_{\geq 0}^{n}: \sum_{j=1}^{n}\left(D_{j}, \lambda\right) x_{j}=(H, \lambda), \quad \forall \lambda \in R(\Sigma)_{\mathbb{Q}}\right\} .
$$

All vertices of $\Delta_{H}$ belong to the sublattice $M_{H} \subset \mathbb{R}^{n}$, where $M_{H}$ is the set of all integral vectors $\left(x_{1}, \ldots, x_{n}\right) \in \mathbb{Z}^{n}$ such that $H$ is linearly equivalent to $\sum_{j=1}^{n} x_{j} D_{j}$.

Let $K_{\text {amp }}(\mathbb{P})$ be the closed ample (or Kähler) cone in $\mathrm{Cl}(\mathbb{P}) \otimes \mathbb{R} \cong H^{2}(\mathbb{P}, \mathbb{R})$ and $K_{\text {eff }}(\mathbb{P})$ the dual to $K_{\text {amp }}(\mathbb{P})$ Mori cone of effective curves in $R(\Sigma)_{\mathbb{R}} \cong H_{2}(\mathbb{P}, \mathbb{R})$. The cone of vectors $\beta=\left(b_{1}, \ldots, b_{n}\right) \in R(\Sigma)_{\mathbb{R}}=R(\Sigma) \otimes \mathbb{R}$ such that $b_{1}, \ldots, b_{n} \geq 0$ will be denoted by $K^{+}(\mathbb{P})$. It is easy to see that $K^{+}(\mathbb{P})$ is always a subcone of the Mori cone $K_{\text {eff }}(\mathbb{P})$.

For any lattice point $\beta=\left(b_{1}, \ldots, b_{n}\right) \in K_{\text {eff }}(\mathbb{P})$, we will construct a simplicial toric variety $\mathbb{P}_{\beta}$ which can be considered as a "naive compactification" of the moduli space of rational maps $\phi: \mathbb{P}^{1} \rightarrow \mathbb{P}$ such that the class $\left[\phi\left(\mathbb{P}^{1}\right)\right] \in H_{2}(\mathbb{P}, \mathbb{R})$ is equal to $\beta$. In the case $\beta=0$, the toric variety $\mathbb{P}_{\beta}$ coincides with $\mathbb{P}$.

First we consider the case $\beta=\left(b_{1}, \ldots, b_{n}\right) \in K^{+}(\mathbb{P})$, i.e., all the $b_{1}, \ldots, b_{n}$ are supposed to be nonnegative. Since $\left(D_{j}, \beta\right)=b_{j}(1 \leq j \leq n)$, we can construct a map $\phi: \mathbb{P}^{1} \rightarrow \mathbb{P}$ with $\left[\phi\left(\mathbb{P}^{1}\right)\right]=\beta$ by choosing $n$ homogeneous binary forms $\phi_{1}(u, v), \ldots, \phi_{n}(u, v) \in \mathbb{C}[u, v]$ such that $\operatorname{deg} \phi_{j}=b_{j}(1 \leq j \leq n)$. Let $\mathbb{C}_{j}(\beta)$ be the space of all homogeneous binary forms of degree $b_{j}\left(\operatorname{dim} \mathbb{C}_{j}(\beta)=b_{j}+1\right)$. 
We set $\mathbb{C}(\beta):=\bigoplus_{j=1}^{n} \mathbb{C}_{j}(\beta)$ and denote by $z_{0}^{(j)}, \ldots, z_{b_{j}}^{(j)}$ the coordinates on $\mathbb{C}_{j}(\beta)$ corresponding to the standard monomial basis of $\mathbb{C}_{j}(\beta)$. We define the action of $G$ on $\mathbb{C}_{j}(\beta)$ as the scalar multiplication by the character $\chi_{j}(1 \leq j \leq n)$. This defines an effective action of $G$ on $\mathbb{C}(\beta)$. If $\chi_{H}=\chi_{1}^{c_{1}} \cdots \chi_{n}^{c_{n}}$ is the character of $G$ corresponding to the class of the ample Cartier divisor $H=\sum_{j=1}^{n} c_{j} D_{j}$ on $\mathbb{P}$, then we define the Morrison-Plesser moduli space $\mathbb{P}_{\beta}$ as the GIT-quotient $\mathbb{C}(\beta) / / G$ with respect to the linearization by $\chi_{H}$ of the structure sheaf on $\mathbb{C}(\beta)$. We define the dense open subset $U(\beta) \subset \mathbb{C}(\beta)$ as the union of all open subsets

$$
U_{i_{1}, \ldots, i_{n-d}}(\sigma)=\left\{z \in \mathbb{C}(\beta): z_{i_{1}}^{\left(j_{1}\right)} z_{i_{2}}^{\left(j_{2}\right)} \cdots z_{i_{n-d}}^{\left(j_{n-d}\right)} \neq 0\right\}
$$

where $\sigma$ runs over all $d$-dimensional cones of $\Sigma,\left\{e_{j_{1}}, e_{j_{2}}, \ldots, e_{j_{n-d}}\right\}$ is the set of all vectors from $\left\{e_{1}, \ldots, e_{n}\right\}$ which do not belong to $\sigma$, and each index $i_{k}(1 \leq k \leq$ $n-d)$ runs independently over all elements of $\left\{0,1, \ldots, b_{k}\right\}$. It is easy to show that the Morrison-Plesser moduli space $\mathbb{P}_{\beta}$ is also the space of orbits $U(\beta) / G$. Moreover, $\mathbb{P}_{\beta}$ is a projective simplicial toric variety of dimension $d+\sum_{j=1}^{n} b_{j}$.

In general, the cone $K^{+}(\mathbb{P})$ is smaller than $K_{\text {eff }}(\mathbb{P})$. In Section 5 we consider such a situation for $\mathbb{P}=\mathbb{F}_{1}$ and show that there exist infinitely many classes $\beta \in$ $K_{\text {eff }}\left(\mathbb{F}_{1}\right) \backslash K^{+}\left(\mathbb{F}_{1}\right)$ which can not be represented by irreducible curves $C \subset \mathbb{F}_{1}$ (see Remark 5.3). Therefore, if one of the coordinates $b_{j}$ of $\beta=\left(b_{1}, \ldots, b_{n}\right)$ is negative, it may happen that there is no rational map $\phi: \mathbb{P}^{1} \rightarrow \mathbb{P}$ such that $\left[\phi\left(\mathbb{P}^{1}\right)\right]=\beta$, but the corresponding Morrison-Plesser moduli space $\mathbb{P}_{\beta}$ (see Definition 3.4 below) is not empty.

Now let $\beta=\left(b_{1}, \ldots, b_{n}\right)$ be an arbitrary lattice point in $K_{\text {eff }}(\mathbb{P})$. For any $j \in$ $\{1, \ldots, n\}$, we define the free abelian group $\mathbb{Z}_{j}(\beta)$ as

$$
\mathbb{Z}_{j}(\beta):= \begin{cases}\mathbb{Z}^{b_{j}+1}, & \text { if } b_{j} \geq 0 \\ 0, & \text { if } b_{j}<0\end{cases}
$$

Using the standard basis of $\mathbb{Z}_{j}(\beta)$, we write each element of $\mathbb{Z}_{j}(\beta)$ as the integral vector $\left(x_{0}^{(j)}, x_{1}^{(j)}, \ldots, x_{b_{j}}^{(j)}\right)$. We set $\mathbb{Z}(\beta):=\bigoplus_{j=1}^{n} \mathbb{Z}_{j}(\beta), \mathbb{R}(\beta):=\mathbb{Z}(\beta) \otimes \mathbb{R}$ and denote by $\mathbb{R}_{\geq 0}(\beta)$ the set of all vectors in $\mathbb{R}(\beta)$ having nonnegative coordinates.

Definition 3.1. Let be $r$ the number of negative coordinates of a lattice point $\beta=\left(b_{1}, \ldots, b_{n}\right) \in K_{\text {eff }}(\mathbb{P})$. Without loss of generality we may assume that $b_{1}, \ldots, b_{n-r} \geq 0$ and $b_{n-r+1}, \ldots, b_{n}<0$. We define the convex set

$$
\Delta_{H}^{\beta}:=\left\{x \in \mathbb{R}_{\geq 0}(\beta): \sum_{j=1}^{n-r}\left(D_{j}, \lambda\right)\left(\sum_{i=0}^{b_{j}} x_{i}^{(j)}\right)=(H, \lambda), \quad \forall \lambda \in R(\Sigma)_{\mathbb{Q}}\right\}
$$


Denote by $M_{H}^{\beta} \subset \mathbb{Z}(\beta)$ the set of all lattice points $x \in \mathbb{Z}(\beta)$ whose coordinates $x_{i}^{(j)}$ satisfy the condition: the divisor

$$
\sum_{j=1}^{n-r}\left(\sum_{i=0}^{b_{j}} x_{i}^{(j)}\right) D_{j}
$$

is linearly equivalent to $H$ up to a linear combination of $D_{n-r+1}, \ldots, D_{n}$.

Proposition 3.2. $\Delta_{H}^{\beta}$ is a compact convex simple polytope having vertices in $M_{H}^{\beta}$. The normal fan $\Sigma_{\beta}$ of the polytope $\Delta_{H}^{\beta}$ does not depend on the choice of the ample divisor $H$.

Proof. In order to see the compactness of $\Delta_{H}^{\beta}$, we remark that there exists a canonical affine linear mapping $\pi_{\beta}: \Delta_{H}^{\beta} \rightarrow \Delta_{H}^{0}=\Delta_{H}$ which replaces every $b_{j}+1$ coordinates $x_{0}^{(j)}, x_{1}^{(j)}, \ldots, x_{b_{j}}^{(j)}$ by their sum $x_{j}=\sum_{i=0}^{b_{j}} x_{i}^{(j)}$. It is clear that $\pi_{\beta}\left(\Delta_{H}^{\beta}\right) \subset \mathbb{R}^{n}$ is a face of $\Delta_{H}^{0}$ defined by the equations $x_{j}=0(n-r \leq j \leq n)$. Therefore $\operatorname{dim} \pi_{\beta}\left(\Delta_{H}^{\beta}\right)=d-r$ if $\pi_{\beta}\left(\Delta_{H}^{\beta}\right)$ is not empty (the latter holds if and only if $e_{n-r}, \ldots, e_{n}$ generate a $r$-dimensional cone in $\Sigma$ ). We observe that the preimage $\pi_{\beta}^{-1}(x)$ of a point in $x \in \pi_{\beta}\left(\Delta_{H}^{\beta}\right)$ is the product of $n-r$ simplices of dimensions $b_{1}, \ldots, b_{n-r}$. Therefore, $\Delta_{H}^{\beta}$ is compact and

$$
\operatorname{dim} \Delta_{H}^{\beta}=d-r+\sum_{j=1}^{n-r} b_{j} .
$$

Now we want to describe all faces of codimension 1 (i.e., facets) of $\Delta_{H}^{\beta}$. It is clear that each facet must be defined by an equation $x_{i}^{(j)}=0$ for some $0 \leq i \leq b_{j}$, $1 \leq j \leq n-r$. However, it is not true in general that every equation $x_{i}^{(j)}=0$ defines a facet. It is easy to show that the equation $x_{i}^{(j)}=0$ defines a facet of $\Delta_{H}^{\beta}$ if and only if either $b_{j}>0$, or if $b_{j}=0$ and $e_{j}, e_{n-r}, \ldots, e_{n}$ generate a $(r+1)$ dimensional cone in $\Sigma$. The $\pi_{\beta}$-image of a vertex $y \in \Delta_{H}^{\beta}$ is a vertex of $\Delta_{H}$. Take an arbitrary vertex $x \in \pi_{\beta}\left(\Delta_{H}^{\beta}\right)$. Since $x$ is a vertex of $\Delta_{H}$, there exists a subset $\left\{i_{1}, \ldots, i_{d}\right\} \subset\{1, \ldots, n\}$ which contains $\{n-r+1, \ldots, n\}$ such that $e_{i_{1}}, \ldots, e_{i_{d}}$ are generators of a $d$-dimensional cone in $\Sigma$ and $x \in \Delta_{H}$ is defined by the conditions $x_{i_{1}}=\cdots=x_{i_{d}}=0$. Since $\Delta_{H}$ is a simple polytope we have $x_{j}>0$ for all $j \notin\left\{i_{1}, \ldots, i_{d}\right\}$ and the equation $x_{j}=\sum_{i=0}^{b_{j}} x_{i}^{(j)}$ defines a $b_{j}$ dimensional simplex with $\left(b_{j}+1\right)$-vertices. In this way, we obtain $\prod_{j}\left(b_{j}+1\right)(j$ runs over $\left.\{1, \ldots, n\} \backslash\left\{i_{1}, \ldots, i_{d}\right\}\right)$ vertices of $\Delta_{H}^{\beta}$ as $\pi_{\beta}$-preimages of $x$. By this method, we get all vertices of $\Delta_{H}^{\beta}$ as $\pi_{\beta}$-preimages of vertices of $\pi_{\beta}\left(\Delta_{H}^{\beta}\right)$. Moreover, one sees that each vertex of $\Delta_{H}^{\beta}$ is contained in exactly $\operatorname{dim} \Delta_{H}^{\beta}$ facets: for each $j \in\left\{i_{1}, \ldots, i_{d}\right\} \backslash\{n-r+1, \ldots, n\}$ we get $b_{j}+1$ facets $x_{j}^{(i)}=0\left(0 \leq i \leq b_{j}\right)$ 
containing a chosen $\pi_{\beta}$-preimage $y$ of $x$ and for each $j \in\{1, \ldots, n\} \backslash\left\{i_{1}, \ldots, i_{d}\right\}$ we get $b_{j}$ facets $x_{j}^{(i)}=0$ containing $y$. Therefore $\Delta_{H}^{\beta}$ is a simple polytope. Since the combinatorial structure of $\Delta_{H}^{\beta}$ is completely determined by $\beta$ and $\Delta_{H}$, it does not depend on the choice of $H$ and the same is true for the normal fan $\Sigma_{\beta}$.

Definition 3.3. Let $\beta=\left(b_{1}, \ldots, b_{n}\right)$ be an arbitrary lattice point in $K_{\text {eff }}(\mathbb{P})$. The projective simplicial toric variety $\mathbb{P}_{\beta}$ associated with the normal fan $\Sigma_{\beta}$ of the polytope $\Delta_{H}^{\beta}$ is called the Morrison-Plesser moduli space corresponding to $\beta \in K_{\text {eff }}(\mathbb{P})$.

It follows from the proof of the last proposition that

$$
\operatorname{dim} \mathbb{P}_{\beta}=\operatorname{dim} \Delta_{H}^{\beta}=d-n+\sum_{j=1}^{n-r}\left(b_{j}+1\right)
$$

if $\mathbb{P}_{\beta}$ is not empty. It is easy to see that the last definition of the Morrison-Plesser moduli space coincides with the previous one in the case $\beta \in K^{+}(\mathbb{P})$. We also remark that $\mathbb{P}_{\beta}$ is nonsingular for all $\beta \in K_{\text {eff }}(\mathbb{P})$ if $\mathbb{P}$ is nonsingular. We will need the following property of the Morrison-Plesser moduli spaces $\mathbb{P}_{\beta}$ :

Proposition 3.4. There exists a canonical surjective homomorphism

$$
\psi_{\beta}: H^{2}(\mathbb{P}, \mathbb{Q}) \rightarrow H^{2}\left(\mathbb{P}_{\beta}, \mathbb{Q}\right)
$$

which is always bijective if $\beta \in K^{+}(\mathbb{P})$.

Proof. Let $\left\{e_{j_{1}}, \ldots, e_{j_{k}}\right\}$ be the set of all generators $e_{j} \in\left\{e_{1}, \ldots, e_{n-r}\right\}$ such that $b_{j}=0$ and $e_{j}, e_{n-r+1}, \ldots, e_{n}$ do not generate a $(r+1)$-dimensional cone in $\Sigma$. We define the subgroup $G_{\beta} \subset G$ to be the common kernel of the characters $\chi_{j_{1}}, \ldots, \chi_{j_{k}}$, i.e.,

$$
G_{\beta}:=\left\{g \in G: \chi_{j_{1}}(g)=\cdots=\chi_{j_{k}}(g)=1\right\} .
$$

Then the simplicial toric variety $\mathbb{P}_{\beta}$ can be obtained as a geometric quotient of an affine space of dimension $\left(b_{1}+1\right)+\cdots+\left(b_{n-r}+1\right)-k$ modulo the linear action of $G_{\beta}$. The embedding $G_{\beta} \hookrightarrow G$ induces the surjective homomorphism of the character groups $\mathrm{Cl}(\mathbb{P}) \rightarrow \mathrm{Cl}\left(\mathbb{P}_{\beta}\right)$. This homomorphism is bijective if $\beta \in K^{+}(\mathbb{P})$, because in the latter case $G=G_{\beta}$. Tensoring by $\mathbb{Q}$, we obtain the canonical surjective homomorphism $\psi_{\beta}: H^{2}(\mathbb{P}, \mathbb{Q}) \rightarrow H^{2}\left(\mathbb{P}_{\beta}, \mathbb{Q}\right)$.

Definition 3.5. Assume that the anticanonical class $-K_{\mathbb{P}}$ of $\mathbb{P}$ is nef, i.e., $\left(-K_{\mathbb{P}}, \beta\right)=$ $\sum_{j=1}^{n} b_{j} \geq 0$ for all $\beta \in K_{\text {eff }}(\mathbb{P})$ and $r$ is the number of negative coordinates of $\beta$, i.e., $b_{1}, \ldots, b_{n-r} \geq 0$ and $b_{n-r+1}, \ldots, b_{n}<0$. By abuse of notations, let us denote 
by $\left[D_{j}\right] \in H^{2}\left(\mathbb{P}_{\beta}, \mathbb{Q}\right)(1 \leq j \leq n)$ also the image of $\left[D_{j}\right] \in H^{2}(\mathbb{P}, \mathbb{Q})$ under $\psi_{\beta}$. Using the multiplication in the cohomology ring $H^{*}\left(\mathbb{P}_{\beta}, \mathbb{Q}\right)$, we define the intersection product

$$
\Phi_{\beta}:=\left(\left[D_{1}\right]+\cdots+\left[D_{n}\right]\right)^{b_{1}+\cdots+b_{n}} \prod_{j=n-r+1}^{n}\left[D_{j}\right]^{-b_{j}-1},
$$

considered as a cohomology class in $H^{2\left(\operatorname{dim} \mathbb{P}_{\beta}-d\right)}\left(\mathbb{P}_{\beta}, \mathbb{Q}\right)$ and call $\Phi_{\beta}$ the MorrisonPlesser class of $\mathbb{P}_{\beta}$.

\section{Toric Residue Mirror Conjecture}

In order to formulate our conjecture, we need some results about Newton polytopes of principal $A$-determinants due to Gelfand, Kapranov and Zelevinsky GKZ.

Let $\Delta \subset M_{\mathbb{R}}$ be a $d$-dimensional polytope with vertices in $M$. Denote by $A$ a finite subset in $\Delta \cap M$ which includes all vertices of $\Delta$.

Definition 4.1. By a triangulation $\mathcal{T}=\left\{\tau_{1}, \ldots, \tau_{k}\right\}$ of $\Delta$ associated with $A$, we mean a decomposition of $\Delta$ into a union of $d$-dimensional simplices $\tau_{1}, \ldots, \tau_{k}$ having vertices in $A$ such that any nonempty intersection $\tau_{i} \cap \tau_{j}$ is a common face of $\tau_{i}$ and $\tau_{j}$. A triangulation $\mathcal{T}$ associated with $A$ is called coherent if there exists a convex piecewise-linear function $\phi: \Delta \rightarrow \mathbb{R}$ whose domains of linearity are precisely the simplices of $\mathcal{T}$.

Definition 4.2. Denote by $\mathbb{R}^{A}$ the space of all real-valued functions on $A$. Let $\mathcal{T}$ be a triangulation of $\Delta$ associated with $A$. The function $\chi_{\mathcal{T}}: A \rightarrow \mathbb{R}$ defined as

$$
m \mapsto \sum_{i: m \in \operatorname{Vert}\left(\tau_{i}\right)} \operatorname{Vol}\left(\tau_{i}\right),
$$

where the sum of the normalized volumes $\operatorname{Vol}\left(\tau_{i}\right)$ runs over all simplices of $\tau_{i} \in$ $\mathcal{T}$ containing $m \in A$ as vertex, is called the characteristic function of $\mathcal{T}$. The secondary polytope $\operatorname{Sec}(A)$ is defined as the convex hull of the vectors $\chi_{\mathcal{T}} \in \mathbb{R}^{A}$, where $\mathcal{T}$ runs over all triangulations of $\Delta$ associated with $A$.

Theorem 4.3 ([GKZ, Chapter 7.1). The secondary polytope $\operatorname{Sec}(A) \subset \mathbb{R}^{A}$ is a $(|A|-d-1)$-dimensional polytope whose vertices are exactly the characteristic functions $\chi_{\mathcal{T}}$ corresponding to all coherent triangulations $\mathcal{T}$ of $\Delta$.

Consider a generic Laurent polynomial

$$
f(t)=\sum_{m \in A} a_{m} t^{m} \in \mathbb{C}\left[t_{1}^{ \pm 1}, \ldots, t_{d}^{ \pm 1}\right] .
$$


The principal $A$-determinant $E_{A}(f)$ is a certain polynomial in $|A|$ independent variables $\left\{a_{m}\right\}_{m \in A}$ with integral coefficients. The following theorem will be very important in the sequel.

Theorem 4.4 ([GKZ], Chapter 10.1, Theorem 1.4). The Newton polytope of $E_{A}(f)$ coincides with the secondary polytope $\operatorname{Sec}(A)$. If $\mathcal{T}=\left\{\tau_{1}, \ldots, \tau_{k}\right\}$ is a coherent triangulation corresponding to some vertex of $\operatorname{Sec}(A)$, then the coefficient at the monomial $\prod_{m \in A} a_{m}^{\chi \mathcal{T}^{(m)}}$ in $E_{A}(f)$ is equal (up to sign) to the product

$$
\prod_{i=1}^{k} \operatorname{Vol}\left(\tau_{i}\right)^{\operatorname{Vol}\left(\tau_{i}\right)}
$$

Recall the notion of a Laurent series of a rational function at a vertex of the Newton polytope of its denominator (see, e.g., GKh or [GKZ, p. 195]).

Definition 4.5. Let $P(a), Q(a) \in \mathbb{C}\left[a_{1}^{ \pm 1}, \ldots, a_{n}^{ \pm 1}\right]$ be two arbitrary Laurent polynomials and let $v \in \mathbb{Z}^{n}$ be a vertex of the Newton polytope of $Q=\sum_{w} c_{w} a^{w}$. We write $Q(a)=c_{v} a^{v}(1+\tilde{Q}(a))$, where

$$
\tilde{Q}(a):=\sum_{w \neq v} \frac{c_{w}}{c_{v}} a^{w-v}
$$

It is easy to see that each Laurent monomial in $a_{1}, \ldots, a_{n}$ appears with nonzero coefficient in $(\tilde{Q}(a))^{i}$ only for finitely many values of $i$. So the expression

$$
\frac{1}{1+\tilde{Q}(a)}=\sum_{i=0}^{\infty}(-1)^{i}(\tilde{Q}(a))^{i}
$$

is well-defined as a Laurent power series in the variables $a_{1}, \ldots, a_{n}$. The product

$$
P(a) \cdot c_{v}^{-1} a^{-v} \cdot\left(1-\tilde{Q}(a)+(\tilde{Q}(a))^{2}-\cdots\right)
$$

is called the Laurent series of the rational function $P(a) / Q(a)$ at the vertex $v$ of the Newton polytope of $Q$.

Now we are able to formulate our Toric Residue Mirror Conjecture:

Conjecture 4.6. Let $\Delta \subset M_{\mathbb{R}}$ be an arbitrary reflexive d-dimensional polytope and $A$ a finite subset in $\Delta \cap M$ containing 0 and all vertices of $\Delta$. Choose any coherent triangulation $\mathcal{T}=\left\{\tau_{1}, \ldots, \tau_{k}\right\}$ of $\Delta$ associated with $A$ such that 0 is a vertex of all the simplices $\tau_{1}, \ldots, \tau_{k}$. Denote by $\mathbb{P}=\mathbb{P}_{\Sigma(\mathcal{T})}$ the simplicial toric variety defined by the fan $\Sigma=\Sigma(\mathcal{T}) \subset M_{\mathbb{R}}$ whose d-dimensional cones are exactly $\sigma_{i}:=\mathbb{R}_{\geq 0} \tau_{i}$ $(1 \leq i \leq k)$. If $A=\left\{v_{0}=0, v_{1}, \ldots, v_{n}\right\}$ and

$$
f(t):=1-\sum_{i=1}^{n} a_{i} t^{v_{i}}
$$


then for any homogeneous polynomial $P\left(x_{1}, \ldots, x_{n}\right) \in \mathbb{Q}\left[x_{1}, \ldots, x_{n}\right]$ of degree $d$ the Laurent expansion of the toric residue

$$
R_{P}(a):=(-1)^{d} \operatorname{Res}_{f}\left(t_{0}^{d} P\left(a_{1} t^{v_{1}}, \ldots, a_{n} t^{v_{n}}\right)\right)
$$

at the vertex $v_{\mathcal{T}} \in \operatorname{Sec}(A)$ corresponding to the coherent triangulations $\mathcal{T}$ coincides with the generating function of intersection numbers

$$
I_{P}(a):=\sum_{\beta \in K_{\mathrm{eff}}(\mathbb{P})} I(P, \beta) a^{\beta},
$$

where the sum runs over all integral points $\beta=\left(b_{1}, \ldots, b_{n}\right)$ of the Mori cone $K_{\text {eff }}(\mathbb{P}), a^{\beta}:=a_{1}^{b_{1}} \cdots a_{n}^{b_{n}}$,

$$
I(P, \beta)=\int_{\mathbb{P}_{\beta}} P\left(\left[D_{1}\right], \ldots,\left[D_{n}\right]\right) \Phi_{\beta}=\left\langle P\left(\left[D_{1}\right], \ldots,\left[D_{n}\right]\right) \Phi_{\beta}\right\rangle_{\beta},
$$

and $\Phi_{\beta} \in H^{2\left(\operatorname{dim} \mathbb{P}_{\beta}-d\right)}\left(\mathbb{P}_{\beta}, \mathbb{Q}\right)$ is the Morrison-Plesser class of $\mathbb{P}_{\beta}$. We assume $I(P, \beta)$ to be zero if $\mathbb{P}_{\beta}$ is empty.

Remark 4.7. Consider any coherent triangulation $\mathcal{T}=\left\{\tau_{1}, \ldots, \tau_{k}\right\}$ of $\Delta$ associated with $A$ such that 0 is a vertex of all simplices and let $v_{\mathcal{T}}$ be the corresponding vertex of the $(n-d)$-dimensional polytope $\operatorname{Sec}(A)$ as above. It is easy to show that the cone $\mathbb{R}_{\geq 0}\left(\operatorname{Sec}(A)-v_{\mathcal{T}}\right)$ can be canonically identified with the Mori cone $K_{\text {eff }}(\mathbb{P}) \subset H_{2}(\mathbb{P}, \mathbb{R})$.

We want to check the statement of our conjecture for the coefficient $I(P, 0)$ of the power series $I_{P}(a)$. For this purpose, we choose a $\Sigma$-piecewise linear function $\varphi$ on $M_{\mathbb{R}}$ corresponding to an ample Cartier divisor $H$ on $\mathbb{P}_{\Sigma}$ and make the substitution $a_{i}=u^{\varphi\left(v_{i}\right)}(1 \leq i \leq n)$, where $u$ is a variable. This substitution is equivalent to the consideration of the 1-parameter family of Laurent polynomials

$$
f(t)=1-\sum_{i=1}^{n} u^{\varphi\left(v_{i}\right)} t^{v_{i}}
$$

depending on $u$. We remark that our substitution transforms $I_{P}(a)$ into a formal power series

$$
I_{P}(u)=\sum_{\beta \in K_{\mathrm{eff}}\left(\mathbb{P}_{\Sigma}\right)} I(P, \beta) u^{(H, \beta)} \in \mathbb{Q}[[u]] .
$$

Since $(H, \beta)>0$ for all nonzero $\beta \in K_{\text {eff }}\left(\mathbb{P}_{\Sigma}\right) \cap R(\Sigma)$, we have

$$
\lim _{u \rightarrow 0} I_{P}(u)=I(P, 0)=\int_{\mathbb{P}} P\left(\left[D_{1}\right], \ldots,\left[D_{n}\right]\right) .
$$

Conjecture 4.6 for $\beta=0$ is equivalent to the following: 
Theorem 4.8. For any homogeneous polynomial $P\left(x_{1}, \ldots, x_{n}\right)$ of degree $d$, one has

$$
(-1)^{d} \lim _{u \rightarrow 0} \operatorname{Res}_{f}\left(t_{0}^{d} P\left(u^{\varphi\left(v_{1}\right)} t^{v_{1}}, \ldots, u^{\varphi\left(v_{n}\right)} t^{v_{n}}\right)=\int_{\mathbb{P}} P\left(\left[D_{1}\right], \ldots,\left[D_{n}\right]\right) .\right.
$$

Proof. Let $\varphi$ be a $\Sigma$-piecewise linear function on $M_{\mathbb{R}}$ corresponding to an ample Cartier divisor on $\mathbb{P}_{\Sigma}$ as above. Without loss of generality we may assume that $\varphi$ is positively defined, i.e., $\varphi \geq 0$ on $M_{\mathbb{R}}$ and $\varphi(x)=0$ for some $x \in M_{\mathbb{R}}$ if and only if $x=0$. Let $S_{\Delta}[u]$ be $\mathbb{C}[u] \otimes_{\mathbb{C}} S_{\Delta}$, which is considered as a graded algebra over the polynomial ring $\mathbb{C}[u]$. We denote by

$$
S_{\varphi}[u]=\bigoplus_{l=0}^{\infty} S_{\varphi}^{l}[u]
$$

the graded $\mathbb{C}[u]$-subalgebra in $S_{\Delta}[u]$ whose $l$-th homogeneous component $S_{\varphi}^{l}[u]$ is spanned as $\mathbb{C}$-vector space by all monomials $u^{r} t_{0}^{l} t^{m}$ such that the lattice point $m$ is contained in $l \Delta$ and $r \geq \varphi(m)$. It is easy to see that the set $S_{\varphi}[u]$ is closed under the multiplication: if $u^{r} t_{0}^{l} t^{m}, u^{r^{\prime}} t_{0}^{l^{\prime}} t^{m^{\prime}} \in S_{\varphi}[u]$, then $u^{r+r^{\prime}} t_{0}^{l+l^{\prime}} t^{m+m^{\prime}} \in S_{\varphi}[u]$, because $r+r^{\prime} \geq \varphi(m)+\varphi\left(m^{\prime}\right) \geq \varphi\left(m+m^{\prime}\right)$.

Let us set $y_{0}:=t_{0}$ and $y_{i}:=-u^{\varphi\left(v_{i}\right)} t_{0} t^{v_{i}}(1 \leq i \leq n)$. By definition, the elements $u, y_{0}, y_{1}, \ldots, y_{n}$ are contained in $S_{\varphi}[u]$. Denote by $\langle u\rangle$ the principal ideal in $S_{\varphi}[u]$ generated by $u$. Using CDS, Proposition 1.2], we obtain the formula

$$
H_{f}=\sum_{J \subset I}(V(J))^{2} \prod_{j \in J} y_{j},
$$

where the sum runs over all subsets $J=\left\{j_{0}, j_{1}, \ldots, j_{d}\right\}$ in $I=\{0,1, \ldots, n\}$ and $V(J)$ is the normalized $d$-dimensional volume of the convex hull $\operatorname{conv}\left(\left\{v_{j_{0}}, v_{j_{1}}, \ldots, v_{j_{d}}\right\}\right)$ (in particular, $V(J)=0$ if dimension of $\operatorname{conv}\left(\left\{v_{j_{0}}, v_{j_{1}}, \ldots, v_{j_{d}}\right\}\right.$ ) is less than $d$ ). If $v_{i_{1}}, \ldots, v_{i_{l}}$ are not vertices of any $d$-dimensional simplex $\tau_{i} \in \mathcal{T}$, then

$$
\begin{gathered}
y_{i_{1}} \cdots y_{i_{l}}=u^{\varphi\left(v_{i_{1}}\right)+\cdots+\varphi\left(v_{i_{l}}\right)} t_{0}^{l} t^{v_{i_{1}}+\cdots+v_{i_{l}}}= \\
=u^{\varphi\left(v_{i_{1}}\right)+\cdots+\varphi\left(v_{i_{l}}\right)-\varphi\left(v_{i_{1}}+\cdots+v_{i_{l}}\right)}\left(u^{\varphi\left(v_{i_{1}}+\cdots+v_{i_{l}}\right)} t_{0}^{l} t^{v_{i_{1}}+\cdots+v_{i_{l}}}\right) \in\langle u\rangle,
\end{gathered}
$$

because the strict convexity of $\varphi$ implies that

$$
\varphi\left(v_{i_{1}}\right)+\cdots+\varphi\left(v_{i_{l}}\right)>\varphi\left(v_{i_{1}}+\cdots+v_{i_{l}}\right) .
$$

Therefore, the Hessian $H_{f}$ can be written as

$$
H_{f}=\sum_{i=1}^{k}\left(\operatorname{Vol}\left(\tau_{i}\right)\right)^{2} \prod_{j: v_{j} \in \tau_{i}} y_{j}+h
$$


for some $h \in\langle u\rangle$. Since every simplex $\tau_{i} \in \mathcal{T}$ contains $v_{0}=0$ and $H_{f}$ is divisible by $y_{0}$, then

$$
H_{f}^{\prime}=\sum_{i=1}^{k}\left(\operatorname{Vol}\left(\tau_{i}\right)\right)^{2} \prod_{j: v_{j} \in \tau_{i}, j \neq 0} y_{j}+h^{\prime}
$$

for some $h^{\prime} \in\langle u\rangle$.

Let $e_{1}, \ldots, e_{d}$ be any basis of the dual lattice $N=\operatorname{Hom}(M, \mathbb{Z})$. Then we can write every monomial $t^{m}(m \in M)$ as product $t_{1}^{m_{1}} \cdots t_{d}^{m_{d}}$, where $m_{i}:=\left\langle m, e_{i}\right\rangle$. Denote by $\mathbb{C}(u)$ the field of rational functions in variable $u$. The toric residue over $\mathbb{C}(u)$ is uniquely determined by $\mathbb{C}[u]$-linear mapping

$$
\operatorname{Res}_{f}[u]: S_{\Delta}^{d}[u] \rightarrow \mathbb{C}(u),
$$

having following two properties:

(1) $\operatorname{Res}_{f}[u]\left(H_{f}^{\prime}\right)=\operatorname{Vol}(\Delta)$;

(2) $\operatorname{Res}_{f}[u]$ vanishes on all $\mathbb{C}[u]$-submodules $F_{i} S_{\Delta}^{d-1}[u] \subset S_{\Delta}^{d}[u](0 \leq i \leq d)$, where

$$
\begin{gathered}
F_{0}:=t_{0} f(t)=y_{0}+\sum_{l=1}^{n} y_{l}, \\
F_{i}:=t_{0} t_{i} \partial f / \partial t_{i}=\sum_{l=1}^{n}\left\langle v_{l}, e_{i}\right\rangle y_{l}, \quad i=1, \ldots, d .
\end{gathered}
$$

Since the toric residue $\operatorname{Res}_{f}$ is the specialization of the $\mathbb{C}[u]$-linear mapping $\operatorname{Res}_{f}[u]$ at the point $u=0, R_{P}(u)=\operatorname{Res}_{f}[u]\left(P\left(y_{1}, \ldots, y_{n}\right)\right) \in \mathbb{Q}(u)$ is regular at the point $u=0$ and

$$
R_{P}(0)=(-1)^{d} \lim _{u \rightarrow 0} \operatorname{Res}_{f}\left(t_{0}^{d} P\left(u^{\varphi\left(v_{1}\right)} t^{v_{1}}, \ldots, u^{\varphi\left(v_{n}\right)} t^{v_{n}}\right)\right.
$$

for any homogeneous polynomial $P\left(y_{1}, \ldots, y_{n}\right) \in \mathbb{Q}\left[y_{1}, \ldots, y_{n}\right]$ of degree $d$.

The cohomology ring $H^{*}(\mathbb{P}, \mathbb{Q})$ of the projective simplicial toric variety $\mathbb{P}$ can be computed as a quotient of the polynomial ring $\mathbb{Q}\left[y_{1}, \ldots, y_{n}\right]$ by the sum of two ideals: $\left\langle F_{1}, \ldots, F_{d}\right\rangle$ and the ideal generated by all monomials $y_{i_{1}} \cdots y_{i_{l}}$ such that $v_{i_{1}}, \ldots, v_{i_{l}}$ are not vertices of any $d$-dimensional simplex $\tau_{i} \in \mathcal{T}$. In this description, the variables $y_{1}, \ldots, y_{n}$ represent the classes of Weil divisors $D_{1}, \ldots, D_{n}$ in $H^{2}(\mathbb{P}, \mathbb{Q})$. Moreover, if $v_{i_{1}}, \ldots, v_{i_{d}}$ are the vertices of a $d$-dimensional simplex $\tau_{i} \in \mathcal{T}$, then the intersection number $\left[D_{i_{1}}\right] \cdots\left[D_{i_{d}}\right]$ equals $1 / \operatorname{Vol}\left(\tau_{i}\right)$.

It follows from (5) and from the property (2) of $\operatorname{Res}_{f}[u]$ that two linear maps

$$
P\left(y_{1}, \ldots, y_{n}\right) \mapsto \int_{\mathbb{P}} P\left(\left[D_{1}\right], \ldots,\left[D_{n}\right]\right)
$$


and

$$
P\left(y_{1}, \ldots, y_{n}\right) \mapsto R_{P}(0)
$$

have the same kernel in the space of homogeneous polynomials of degree $d$. Therefore, in order to identify these linear maps, it is sufficient to compare their values on the special polynomial

$$
\widetilde{P}\left(y_{1}, \ldots, y_{n}\right):=\sum_{i=1}^{k}\left(\operatorname{Vol}\left(\tau_{i}\right)\right)^{2} \prod_{j: v_{j} \in \tau_{i}, j \neq 0} y_{j} .
$$

By the property (1) of $\operatorname{Res}_{f}[u]$ and (白), we obtain $R_{\widetilde{P}}(0)=\operatorname{Vol}(\Delta)$. On the other hand,

$$
\begin{gathered}
\int_{\mathbb{P}} \widetilde{P}\left(\left[D_{1}\right], \ldots,\left[D_{n}\right]\right)=\sum_{i=1}^{k}\left(\operatorname{Vol}\left(\tau_{i}\right)\right)^{2} \int_{\mathbb{P}} \prod_{j: v_{j} \in \tau_{i}, j \neq 0}\left[D_{j}\right] \\
=\sum_{i=1}^{k}\left(\operatorname{Vol}\left(\tau_{i}\right)\right)^{2} \frac{1}{\operatorname{Vol}\left(\tau_{i}\right)}=\sum_{i=1}^{k} \operatorname{Vol}\left(\tau_{i}\right)=\operatorname{Vol}(\Delta) .
\end{gathered}
$$

This finishes the proof.

Remark 4.9. We remark that the number $\operatorname{Vol}(\Delta)$ equals the stringy Euler number $e_{\mathrm{st}}(\mathbb{P})$ of the simplicial toric variety $\mathbb{P} \mathbb{B a t 3}$. It is known that the usual Euler number $e(\mathbb{P})$ equals $k$ (the number of $d$-dimensional cones in $\Sigma$ ). The top Chern class of $\mathbb{P}$ is represented in the cohomology ring $H^{*}(\mathbb{P}, \mathbb{Q})$ by the polynomial

$$
C_{d}\left(y_{1}, \ldots, y_{n}\right):=\sum_{i=1}^{k} \operatorname{Vol}\left(\tau_{i}\right) \prod_{j: v_{j} \in \tau_{i}, j \neq 0} y_{j} .
$$

In the proof of the last theorem, we have shown that the Hessian $H_{f}^{\prime}$ specializes at $u=0$ to the polynomial $\widetilde{P}\left(y_{1}, \ldots, y_{n}\right)$ which represents the stringy top Chern class of $\mathbb{P}$.

\section{Hirzebruch SuRface $\mathbb{F}_{1}$}

We consider below a simplest example which illustrates many interesting ingredients of our conjecture.

Let $M \cong \mathbb{Z}^{2}$ and $\Delta \subset M_{\mathbb{R}} \cong \mathbb{R}^{2}$ be a reflexive polytope with the vertices

$$
v_{1}=(-1,1), \quad v_{2}=(0,-1), \quad v_{3}=(1,0), \quad v_{4}=(0,1) .
$$

Denote by $A$ the set of points $\left\{0, v_{1}, \ldots, v_{4}\right\}$. Take a coherent triangulation $\mathcal{T}=$ $\left\{\tau_{1}, \ldots, \tau_{4}\right\}$ of $\Delta$ associated with $A$ such that 0 is a vertex of every $\tau_{i}$. The surface 
$\mathbb{F}_{1}$ is a toric variety $\mathbb{P}_{\Sigma}$ defined by the fan $\Sigma=\Sigma(\mathcal{T}) \subset M_{\mathbb{R}}$ (see Figure 国) whose 1-dimensional cones are generated by $v_{i}$. Take a generic Laurent polynomial

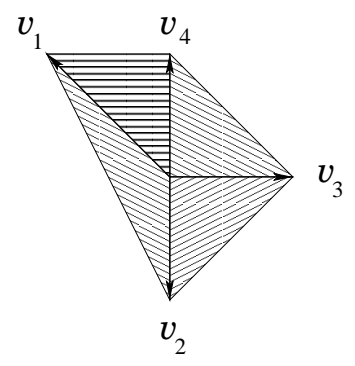

FiguRe 1. Fan $\Sigma$ for the Hirzebruch surface $\mathbb{P}_{\Sigma}=\mathbb{F}_{1}$

$$
f(t)=1-a_{1} t^{v_{1}}-a_{2} t^{v_{2}}-a_{3} t^{v_{3}}-a_{4} t^{v_{4}}=1-a_{1} t_{1}^{-1} t_{2}-a_{2} t_{2}^{-1}-a_{3} t_{1}-a_{4} t_{2}
$$

with support in $\Delta$.

Proposition 5.1. The principal A-determinant of $f(t)$ is

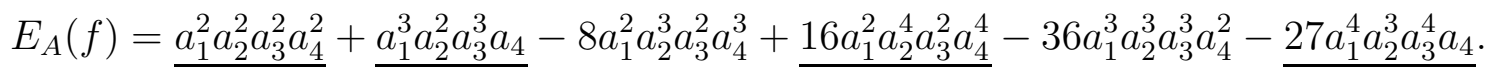

The underlined terms are in one-to-one correspondence with the vertices of the secondary polytope $\operatorname{Sec}(A)$. Moreover, the vertex $v_{\mathcal{T}}$ corresponding to the triangulation $\mathcal{T}$ is related with the monomial $a_{1}^{2} a_{2}^{2} a_{3}^{2} a_{4}^{2}$.

Proof. It is easy to find all coherent triangulations of $\Delta$ (see Figure 2). By knowing the coherent triangulations, using Theorem 4.4, we can compute (up to sign) the terms of $E_{A}(f)$ corresponding to the vertices of $\operatorname{Sec}(A)$ :

$$
a_{1}^{2} a_{2}^{2} a_{3}^{2} a_{4}^{2}, a_{1}^{3} a_{2}^{2} a_{3}^{3} a_{4}, 16 a_{1}^{2} a_{2}^{4} a_{3}^{2} a_{4}^{4}, 27 a_{1}^{4} a_{2}^{3} a_{3}^{4} a_{4} .
$$

In order to find the other terms, we compute (up to multiplication by monomial) the discriminant $D_{A}(f)$, i.e., the set of those coefficients $\left\{a_{i}\right\}$ of $f(t)$ such that the system

$$
f(t)=t_{1} \frac{\partial f}{\partial t_{1}}(t)=t_{2} \frac{\partial f}{\partial t_{2}}(t)=0
$$

has a solution in the torus $\mathbb{T} \cong\left(\mathbb{C}^{*}\right)^{2}$. If we define

$$
Z_{1}:=t_{1}^{-1} t_{2}, Z_{2}:=t_{2}^{-1}, Z_{3}:=t_{1}, Z_{4}:=t_{2},
$$

then we obtain $Z_{4}=Z_{1} Z_{3}, Z_{2} Z_{4}=1$ and previous three equations can be rewritten as

$$
\begin{array}{r}
1-a_{1} Z_{1}-\cdots-a_{4} Z_{4}=0, a_{1} Z_{1}-a_{3} Z_{3}=0 \\
a_{2} Z_{2}-a_{1} Z_{1}-a_{4} Z_{4}=0 .
\end{array}
$$


Excluding $Z_{3}$ and $Z_{4}$, we get

$$
1-a_{1} Z_{1}-2 a_{2} Z_{2}=0, a_{3}\left(a_{2} Z_{2}-a_{1} Z_{1}\right)=a_{1} a_{4} Z_{1}^{2}, Z_{2}\left(a_{2} Z_{2}-a_{1} Z_{1}\right)=a_{4} .
$$

The last system is equivalent to two homogeneous equations

$$
a_{3}\left(a_{1} Z_{1}+2 a_{2} Z_{2}\right)\left(a_{2} Z_{2}-a_{1} Z_{1}\right)=a_{1} a_{4} Z_{1}^{2}, Z_{2}\left(a_{2} Z_{2}-a_{1} Z_{1}\right)=a_{4}\left(a_{1} Z_{1}+2 a_{2} Z_{2}\right)^{2} .
$$

Computing the resultant of two polynomials, we get the discriminant

$$
D_{A}(f)=a_{4}+a_{1} a_{3}-8 a_{2} a_{4}^{2}+16 a_{2}^{2} a_{4}^{3}-36 a_{1} a_{2} a_{3} a_{4}-27 a_{1}^{2} a_{2} a_{3}^{2} .
$$

We remark that the Newton polytope of $E_{A}(f)$ (secondary polytope) is obtained from the Newton polytope of $D_{A}(f)$ via a shift by vector $(2,2,2,1)$. So the multiplication of $D_{A}(f)$ by $a_{1}^{2} a_{2}^{2} a_{3}^{2} a_{4}$ yields a polynomial having $\operatorname{Sec}(A)$ as its Newton polytope $\operatorname{Sec}(A)$. Since $D_{A}(f)$ divides $E_{A}(f)$ (see Remark 2.8), we conclude that $a_{1}^{2} a_{2}^{2} a_{3}^{2} a_{4} D_{A}(f)$ is exactly $E_{A}(f)$.

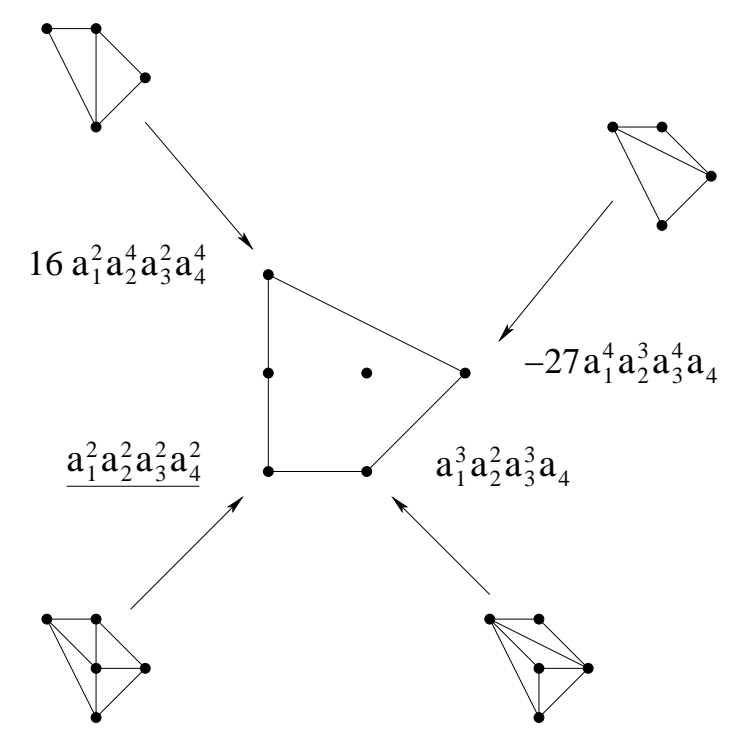

FiguRE 2. Secondary polytope $\operatorname{Sec}(A)$

For the case $\mathbb{P}=\mathbb{F}_{1}$, Conjecture 4.6 can be reformulated as follows:

Conjecture 5.2. Let $D_{1}, \ldots, D_{4}$ be the torus-invariant divisors on $\mathbb{P}_{\Sigma}$ corresponding to the vectors $v_{1}, \ldots, v_{4}$ respectively. Fix a homogeneous polynomial $P\left(x_{1}, \ldots, x_{4}\right)$ of degree two in $\mathbb{Q}\left[x_{1}, \ldots, x_{4}\right]$. Then the series expansion of the toric residue

$$
R_{P}\left(a_{1}, \ldots, a_{4}\right)=\operatorname{Res}_{f}\left(t_{0}^{2} P\left(a_{1} t^{v_{1}}, \ldots, a_{4} t^{v_{1}}\right)\right)
$$


at the vertex $v_{\mathcal{T}} \in \operatorname{Sec}(A)$ defined by the triangulation $\mathcal{T}$ coincides with the generating function of the intersection numbers

$$
I_{P}\left(a_{1}, \ldots, a_{4}\right)=\sum_{\beta \in K_{\mathrm{eff}}\left(\mathbb{P}_{\Sigma}\right)}\left\langle P\left(\left[D_{1}\right], \ldots,\left[D_{4}\right]\right) \Phi_{\beta}\right\rangle_{\beta} a^{\beta},
$$

where $\Phi_{\beta}=\left(\left[D_{1}\right]+\cdots+\left[D_{4}\right]\right)^{b_{1}+\cdots+b_{4}} \prod_{i: b_{i}<0}\left[D_{i}\right]^{-b_{i}-1}, a^{\beta}=a_{1}^{b_{1}} \cdots a_{4}^{b_{4}}$.

It is possible to compute explicitly both the generating function for intersection numbers and the toric residue. In this way, we check the equality of Conjecture 5.2 by direct calculation. We will omit the details of this calculation and sketch only ideas of how it can be done.

First, we remark that the Mori cone $K_{\text {eff }}\left(\mathbb{P}_{\Sigma}\right) \subset R(\Sigma)_{\mathbb{R}}$ is spanned by two elements

$$
l^{(1)}=(1,0,1,-1), \quad l^{(2)}=(0,1,0,1),
$$

i.e., $\beta$ runs over all lattice points

$$
\left(b_{1}, \ldots, b_{4}\right)=\lambda_{1}(1,0,1,-1)+\lambda_{2}(0,1,0,1)=\left(\lambda_{1}, \lambda_{2}, \lambda_{1}, \lambda_{2}-\lambda_{1}\right), \quad \lambda_{1}, \lambda_{2} \geq 0 .
$$

The dual to $K_{\text {eff }}\left(\mathbb{P}_{\Sigma}\right)$ Kähler cone is generated by the classes of $D_{1}$ and $D_{2}$. There are two independent linear relations between the classes of torus-invariant divisors $D_{1}, D_{2}, D_{3}, D_{4}$ :

$$
\left[D_{1}\right]-\left[D_{3}\right]=0, \quad\left[D_{1}\right]-\left[D_{2}\right]+\left[D_{4}\right]=0 .
$$

We can consider the classes of $D_{1}, D_{2}$ as generators of $H^{2}\left(\mathbb{P}_{\Sigma}, \mathbb{Z}\right)$ and put

$$
y_{1}:=a^{l^{(1)}}=\frac{a_{1} a_{3}}{a_{4}}, \quad y_{2}:=a^{l^{(2)}}=a_{2} a_{4}
$$

so that $a^{\beta}=a_{1}^{b_{1}} \cdots a_{4}^{b_{4}}$ is equal to $y_{1}^{\lambda_{1}} y_{2}^{\lambda_{2}}$. Substituting above linear relations, we get

$$
P\left(\left[D_{1}\right], \ldots,\left[D_{4}\right]\right)=P\left(\left[D_{1}\right],\left[D_{2}\right],\left[D_{1}\right],\left[D_{2}\right]-\left[D_{1}\right]\right) .
$$

By linearity, it is sufficient to compute the generating functions

$$
I_{x_{1}^{i} x_{2}^{2-i}}\left(y_{1}, y_{2}\right)=\sum_{\lambda_{1}, \lambda_{2} \geq 0}\left\langle\left[D_{1}\right]^{i}\left[D_{2}\right]^{2-i} \Phi_{\beta}\right\rangle_{\beta} y_{1}^{\lambda_{1}} y_{2}^{\lambda_{2}}, \quad i=0,1,2 .
$$

We divide the Mori cone of $\mathbb{P}_{\Sigma}$ into two parts (see Figure 3):

(1) $\lambda_{1}>\lambda_{2} \geq 0$

(2) $\lambda_{2} \geq \lambda_{1} \geq 0$.

For each of the part of the Mori cone we find the coefficients of the generating function $I_{x_{1}^{i} x_{2}^{2-i}}\left(y_{1}, y_{2}\right)$.

CASE 1. If $\lambda_{1}>\lambda_{2} \geq 0$, then the last coordinate $b_{4}$ of

$$
\beta=\left(b_{1}, \ldots, b_{4}\right)=\left(\lambda_{1}, \lambda_{2}, \lambda_{1}, \lambda_{2}-\lambda_{1}\right)
$$




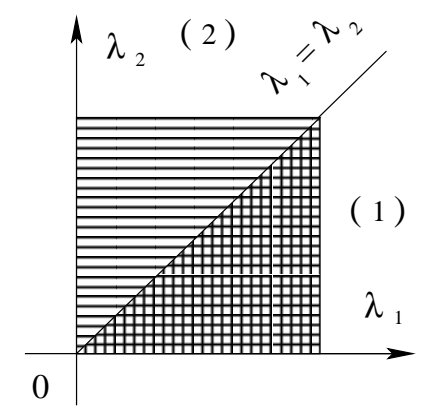

Figure 3. Two parts of the Mori cone of $\mathbb{P}_{\Sigma}$

is negative. By definition of the Morrison-Plesser moduli spaces $\mathbb{P}_{\beta}$ and the MorrisonPlesser classes $\Phi_{\beta}$, we obtain $\mathbb{P}_{\beta}=\mathbb{P}^{2 \lambda_{1}+1} \times \mathbb{P}^{\lambda_{2}}$ and

$$
\Phi_{\beta}=\left(\left[D_{1}\right]+2\left[D_{2}\right]\right)^{\lambda_{1}+2 \lambda_{2}}\left(\left[D_{2}\right]-\left[D_{1}\right]\right)^{\lambda_{1}-\lambda_{2}-1} .
$$

The intersection theory on $\mathbb{P}_{\beta}$ shows immediately that

$$
\left\langle\left[D_{1}\right]^{l_{1}}\left[D_{2}\right]^{l_{2}}\right\rangle_{\beta}= \begin{cases}1, & l_{1}=2 \lambda_{1}+1, \quad l_{2}=\lambda_{2} \\ 0, & \text { otherwise }\end{cases}
$$

So we obtain

$$
\begin{aligned}
\left\langle\left[D_{1}\right]^{i}\left[D_{2}\right]^{2-i} \Phi_{\beta}\right\rangle_{\beta} & =\left\langle\left[D_{1}\right]^{i}\left[D_{2}\right]^{2-i}\left(\left[D_{1}\right]+2\left[D_{2}\right]\right)^{\lambda_{1}+2 \lambda_{2}}\left(\left[D_{2}\right]-\left[D_{1}\right]\right)^{\lambda_{1}-\lambda_{2}-1}\right\rangle_{\beta} \\
= & \sum_{k \geq 0}(-1)^{k} 2^{2 \lambda_{2}-\lambda_{1}+k-1+i}\left(\begin{array}{c}
\lambda_{1}-\lambda_{2}-1 \\
k
\end{array}\right)\left(\begin{array}{c}
\lambda_{1}+2 \lambda_{2} \\
2 \lambda_{1}-k+1-i
\end{array}\right) .
\end{aligned}
$$

Remark 5.3. We remark that there is no irreducible curve $C \subset \mathbb{F}_{1}$ such that $[C]=$ $\beta=\left(b_{1}, \ldots, b_{4}\right)$, if $b_{4}<0$, and $b_{2}>0$. Indeed, if $C \subset \mathbb{F}_{1}$ were such a curve, then $b_{4}=\left(C, D_{4}\right)<0$ would imply that $[C]$ is proportional to $\left[D_{4}\right]$ (the latter contradicts $b_{2}=\left(C, D_{2}\right)>0$ because of $\left.\left(D_{4}, D_{2}\right)=0\right)$. On the other hand, the corresponding Morrison-Plesser moduli spaces $\mathbb{P}_{\beta}=\mathbb{P}^{2 b_{1}+1} \times \mathbb{P}^{b_{2}}$ are always nonempty.

CASE 2. Let $\lambda_{2} \geq \lambda_{1} \geq 0$. Then the Morrison-Plesser moduli spaces $\mathbb{P}_{\beta}$ are toric varieties of dimension $\lambda_{1}+2 \lambda_{2}+2$. The Morrison-Plesser class is equal to $\Phi_{\beta}=\left(\left[D_{1}\right]+2\left[D_{2}\right]\right)^{\lambda_{1}+2 \lambda_{2}}$ and the coefficients of the series $I_{x_{1}^{i} x_{2}^{2-i}}\left(y_{1}, y_{2}\right)$ are the intersection numbers

$$
\left\langle\left[D_{1}\right]^{i}\left[D_{2}\right]^{2-i} \Phi_{\beta}\right\rangle_{\beta}=\left\langle\left[D_{1}\right]^{i}\left[D_{2}\right]^{2-i}\left(\left[D_{1}\right]+2\left[D_{2}\right]\right)^{\lambda_{1}+2 \lambda_{2}}\right\rangle_{\beta} .
$$


These numbers can be computed directly using the intersection theory on the Morrison-Plesser moduli spaces $\mathbb{P}_{\beta}$. We omit the details of the proof. It is remarkable that the obtained formula

$$
\left\langle\left[D_{1}\right]^{i}\left[D_{2}\right]^{2-i} \Phi_{\beta}\right\rangle_{\beta}=\sum_{k \geq 0} 2^{2 \lambda_{2}-\lambda_{1}+k-1+i}\left(\begin{array}{c}
\lambda_{2}-\lambda_{1}+k \\
k
\end{array}\right)\left(\begin{array}{c}
\lambda_{1}+2 \lambda_{2} \\
2 \lambda_{1}-k+1-i
\end{array}\right)
$$

can be found from the formula obtained in Case 1 by the analytic continuation of the binomial coefficients:

$$
\left(\begin{array}{c}
-m \\
n
\end{array}\right):=(-1)^{n}\left(\begin{array}{c}
m+n-1 \\
n
\end{array}\right), \quad n=0, \pm 1, \pm 2, \ldots
$$

for any positive integer $m$.

Summarizing all the considered cases, we obtain the generating function of the intersection numbers in the form

$$
\begin{aligned}
& I_{x_{1}^{i} x_{2}^{2-i}}\left(y_{1}, y_{2}\right)= \\
= & \sum_{\lambda_{1}, \lambda_{2} \geq 0}\left(\sum_{k \geq 0}(-1)^{k} 2^{2 \lambda_{2}-\lambda_{1}+k-1+i}\left(\begin{array}{c}
\lambda_{1}-\lambda_{2}-1 \\
k
\end{array}\right)\left(\begin{array}{c}
\lambda_{1}+2 \lambda_{2} \\
2 \lambda_{1}-k+1-i
\end{array}\right)\right) y_{1}^{\lambda_{1}} y_{2}^{\lambda_{2}} .
\end{aligned}
$$

In order to compare this series with toric residues, we use the following integral representation:

$$
I_{x_{1}^{i} x_{2}^{2-i}}\left(y_{1}, y_{2}\right)=\sum_{\lambda_{1}, \lambda_{2} \geq 0}\left(\frac{1}{(2 \pi i)^{2}} \int_{\gamma} \frac{u_{0}^{i} u_{1}^{2-i}\left(u_{1}-u_{0}\right)^{\lambda_{1}-\lambda_{2}-1}\left(u_{0}+2 u_{1}\right)^{\lambda_{1}+\lambda_{2}} d u_{0} \wedge d u_{1}}{u_{0}^{2 \lambda_{1}+2} u_{1}^{\lambda_{2}+1}}\right) y_{1}^{\lambda_{1}} y_{2}^{\lambda_{2}},
$$

where $\gamma=\left\{\left(u_{0}, u_{1}\right) \in \mathbb{C}^{2}:\left|u_{0}\right|=\varepsilon_{1},\left|u_{1}\right|=\varepsilon_{2}\right\}, \varepsilon_{1}, \varepsilon_{2}>0$. Changing the order of summation and integration, we get

$$
I_{x_{1}^{i} x_{2}^{2-i}}\left(y_{1}, y_{2}\right)=\frac{1}{(2 \pi i)^{2}} \int_{\gamma} \frac{u_{0}^{i} u_{1}^{2-i} d u_{0} \wedge d u_{1}}{\left(u_{0}^{2}-\left(u_{0}+2 u_{1}\right)\left(u_{1}-u_{0}\right) y_{1}\right)\left(u_{1}\left(u_{1}-u_{0}\right)-\left(u_{0}+2 u_{1}\right)^{2} y_{2}\right)} .
$$

This integral can be computed directly. We write down the obtained rational functions $I_{Q}\left(y_{1}, y_{2}\right)$ together with some first terms of their expansions:

- $Q\left(x_{1}, x_{2}\right)=x_{2}^{2}$.

$$
\begin{aligned}
& \frac{1+y_{1}+4 y_{2}+3 y_{1} y_{2}}{1+y_{1}-8 y_{2}+16 y_{2}^{2}-36 y_{1} y_{2}-27 y_{1}^{2} y_{2}}=1+12 y_{2}+27 y_{1} y_{2}+80 y_{2}^{2} \\
& \quad+568 y_{1} y_{2}^{2}+448 y_{2}^{3}+728 y_{1}^{2} y_{2}^{2}+6544 y_{1} y_{2}^{3}+2304 y_{2}^{4}+y_{1}^{3} y_{2}^{2}+21888 y_{1}^{2} y_{2}^{3}+\cdots
\end{aligned}
$$


- $Q\left(x_{1}, x_{2}\right)=x_{1} x_{2}$ :

$$
\begin{aligned}
& \frac{1+y_{1}-4 y_{2}-6 y_{1} y_{2}}{1+y_{1}-8 y_{2}+16 y_{2}^{2}-36 y_{1} y_{2}-27 y_{1}^{2} y_{2}}=1+4 y_{2}+26 y_{1} y_{2}+16 y_{2}^{2}+y_{1}^{2} y_{2} \\
& +336 y_{1} y_{2}^{2}+64 y_{2}^{3}-y_{1}^{3} y_{2}+716 y_{1}^{2} y_{2}^{2}+2784 y_{1} y_{2}^{3}+256 y_{2}^{4}+y_{1}^{4} y_{2}+14 y_{1}^{3} y_{2}^{2}+\cdots
\end{aligned}
$$

- $Q\left(x_{1}, x_{2}\right)=x_{1}^{2}$ :

$$
\begin{aligned}
& \frac{y_{1}\left(1+12 y_{2}\right)}{1+y_{1}-8 y_{2}+16 y_{2}^{2}-36 y_{1} y_{2}-27 y_{1}^{2} y_{2}}=y_{1}-y_{1}^{2}+20 y_{1} y_{2}+y_{1}^{3}+8 y_{1}^{2} y_{2} \\
& +144 y_{1} y_{2}^{2}-y_{1}^{4}-9 y_{1}^{3} y_{2}+656 y_{1}^{2} y_{2}^{2}+832 y_{1} y_{2}^{3}+y_{1}^{5}+10 y_{1}^{4} y_{2}+84 y_{1}^{3} y_{2}^{2}+\cdots
\end{aligned}
$$

These rational functions can be identified with the toric residues $R_{x_{1}^{i} x_{2}^{2-i}}\left(y_{1}, y_{2}\right)$ $(i=0,1,2)$ which one computes, for example, by the method from Section 9. This verifies Conjecture 5.2 directly.

\section{TORIC RESIDUE AND FLOP}

In this section, we consider a simplest reflexive polytope $\Delta$ which has two different coherent triangulations such that 0 is a vertex of all simplices. These triangulations correspond to two different vertices of the secondary polytope. We compute the corresponding expansions of the toric residues at each of these vertices.

Denote by $A$ the union of the origin $v_{0}=(0,0,0)$ in $M \cong \mathbb{Z}^{3}$ together with the points

$$
v_{1}=(1,0,0), v_{2}=(0,1,0), v_{3}=(-1,-1,0), v_{4}=(0,0,1), v_{5}=(1,1,-1) .
$$

Then $\Delta:=\operatorname{conv}(A) \subset M_{\mathbb{R}} \cong \mathbb{R}^{3}$ is the reflexive polytope. Let

$$
f(t)=1-\sum_{i=1}^{5} a_{i} t^{v_{i}}=1-a_{1} t_{1}-a_{2} t_{2}-a_{3} t_{1}^{-1} t_{2}^{-1}-a_{4} t_{3}-a_{5} t_{1} t_{2} t_{3}^{-1}
$$

be a generic Laurent polynomial.

Proposition 6.1. The principal A-determinant of $f(t)$ equals

$$
\begin{gathered}
E_{A}(f)=\frac{a_{1}^{4} a_{2}^{4} a_{3}^{4} a_{4}^{3} a_{5}^{3}}{-54 a_{1}^{5} a_{2}^{5} a_{3}^{5} a_{4}^{3} a_{5}^{3}-a_{1}^{3} a_{2}^{3} a_{3}^{4} a_{4}^{4} a_{5}^{4}}+\underline{729 a_{1}^{6} a_{2}^{6} a_{3}^{6} a_{4}^{3} a_{5}^{3}} \\
+54 a_{1}^{3} a_{2}^{3} a_{3}^{5} a_{4}^{5} a_{5}^{5}-2187 a_{1}^{5} a_{2}^{5} a_{3}^{6} a_{4}^{4} a_{5}^{4}+2187 a_{1}^{4} a_{2}^{4} a_{3}^{6} a_{4}^{5} a_{5}^{5}-\underline{729 a_{1}^{3} a_{2}^{3} a_{3}^{6} a_{4}^{6} a_{5}^{6},}
\end{gathered}
$$

where the terms corresponding to the vertices of the secondary polytope $\operatorname{Sec}(A)$ are underlined.

Proof. The idea of the proof is the same as in Proposition 5.1. The terms corresponding to the vertices of the polytope $\operatorname{Sec}(A)$ can be easily found (up to sign) from the coherent triangulations of the polytope $\Delta$ :

$$
a_{1}^{4} a_{2}^{4} a_{3}^{4} a_{4}^{3} a_{5}^{3}, a_{1}^{3} a_{2}^{3} a_{3}^{4} a_{4}^{4} a_{5}^{4}, 729 a_{1}^{6} a_{2}^{6} a_{3}^{6} a_{4}^{3} a_{5}^{3}, 729 a_{1}^{3} a_{2}^{3} a_{3}^{6} a_{4}^{6} a_{5}^{6} .
$$


The Laurent polynomial $f$ is $\Delta$-regular, i.e., $E_{A}(f) \neq 0$, if and only if the equations

$$
f(t)=t_{1} \frac{\partial f}{\partial t_{1}}(t)=t_{2} \frac{\partial f}{\partial t_{2}}(t)=t_{3} \frac{\partial f}{\partial t_{3}}(t)=0
$$

have no solution in the compactification $\mathbb{P}_{\Delta}$ of the torus $\mathbb{T} \cong\left(\mathbb{C}^{*}\right)^{3}$. If we put

$$
Z_{1}:=t_{1}, Z_{2}:=t_{2}, Z_{3}:=t_{1}^{-1} t_{2}^{-1}, Z_{4}:=t_{3}, Z_{5}:=t_{1} t_{2} t_{3}^{-1},
$$

then the $Z_{0}$-homogenization of the last system is equivalent to

$$
\begin{array}{r}
Z_{0}-a_{1} Z_{1}-\cdots-a_{5} Z_{5}=a_{4} Z_{4}-a_{5} Z_{5}=a_{1} Z_{1}-a_{3} Z_{3}+a_{5} Z_{5} \\
=a_{2} Z_{2}-a_{3} Z_{3}+a_{5} Z_{5}=0 ; Z_{1} Z_{2} Z_{3}=Z_{0}^{3}, Z_{1} Z_{2}=Z_{4} Z_{5}
\end{array}
$$

By excluding $Z_{1}, Z_{2}, Z_{4}$, we obtain

$$
Z_{0}-3 a_{3} Z_{3}=\left(a_{3} Z_{3}-a_{5} Z_{5}\right)^{2} Z_{3}-a_{1} a_{2} Z_{0}^{3}=a_{4}\left(a_{3} Z_{3}-a_{5} Z_{5}\right)^{2}-a_{1} a_{2} a_{5} Z_{5}^{2}=0 .
$$

Hence, $\Delta$-regularity of $f$ is equivalent to nonvanishing of the resultant $R\left(g_{1}, g_{2}\right)$ of two homogeneous forms

$$
g_{1}=\left(a_{3} Z_{3}-a_{5} Z_{5}\right)^{2} Z_{3}-27 a_{1} a_{2} a_{3}^{3} Z_{3}^{3}, g_{2}=a_{4}\left(a_{3} Z_{3}-a_{5} Z_{5}\right)^{2}-a_{1} a_{2} a_{5} Z_{5}^{2} .
$$

By direct computation, we obtain that $R\left(g_{1}, g_{2}\right)$ splits into product of two irreducible divisors of multiplicity 1 . Moreover, it is straightforward to see that the difference between the Newton polytopes of $R\left(g_{1}, g_{2}\right)$ and $E_{A}(f)$ is the vector $(1,1,0,3,0)$. Since $R\left(g_{1}, g_{2}\right)$ and $E_{A}(f)$ have the same set of irreducible divisors $D_{A \cap \Gamma}(f), \Gamma \subseteq \Delta$ (see Remark 2.8), we see that the multiplication of $R\left(g_{1}, g_{2}\right)$ by $-a_{1} a_{2} a_{4}^{3}$ yields $E_{A}(f)$.

The toric residue in the following statement can be computed using, for example, the method from Section 9 .

Proposition 6.2. Let $P\left(x_{1}, x_{2}, x_{3}, x_{4}\right)=x_{1} x_{2} x_{4} \in \mathbb{Q}\left[x_{1}, \ldots, x_{4}\right]$ be the input polynomial. Then the corresponding toric residue

$$
R_{x_{1} x_{2} x_{4}}\left(a_{1}, \ldots, a_{5}\right)=-\operatorname{Res}_{f}\left(t_{0}^{3}\left(a_{1} t_{1}\right)\left(a_{2} t_{2}\right)\left(a_{4} t_{3}\right)\right)
$$

can be expressed as a quotient

$$
R_{x_{1} x_{2} x_{4}}\left(a_{1}, \ldots, a_{5}\right)=\left(a_{1}^{4} a_{2}^{4} a_{3}^{4} a_{4}^{3} a_{5}^{3}-27 a_{1}^{5} a_{2}^{5} a_{3}^{5} a_{4}^{3} a_{5}^{3}-81 a_{1}^{4} a_{2}^{4} a_{3}^{5} a_{4}^{4} a_{5}^{4}\right) / E_{A}(f) .
$$

There are two triangulations related with the vectors $v_{1}, \ldots, v_{5}$ with the property that their maximal-dimensional simplices contain 0 (see Figure 『). Namely,

$$
\mathcal{T}_{1}=\left\{\tau_{124}, \tau_{125}, \tau_{134}, \tau_{135}, \tau_{235}, \tau_{234}\right\}
$$

and

$$
\mathcal{T}_{2}=\left\{\tau_{145}, \tau_{245}, \tau_{134}, \tau_{135}, \tau_{235}, \tau_{234}\right\},
$$

here $\tau_{124}$ means that this simplex is generated by $0, v_{1}, v_{2}, v_{4}$, etc. Let $\Sigma_{1}=$ $\Sigma\left(\mathcal{T}_{1}\right) \subset M_{\mathbb{R}}\left(\Sigma_{2}=\Sigma\left(\mathcal{T}_{2}\right) \subset M_{\mathbb{R}}\right)$ be the fan whose $d$-dimensional simplices are 

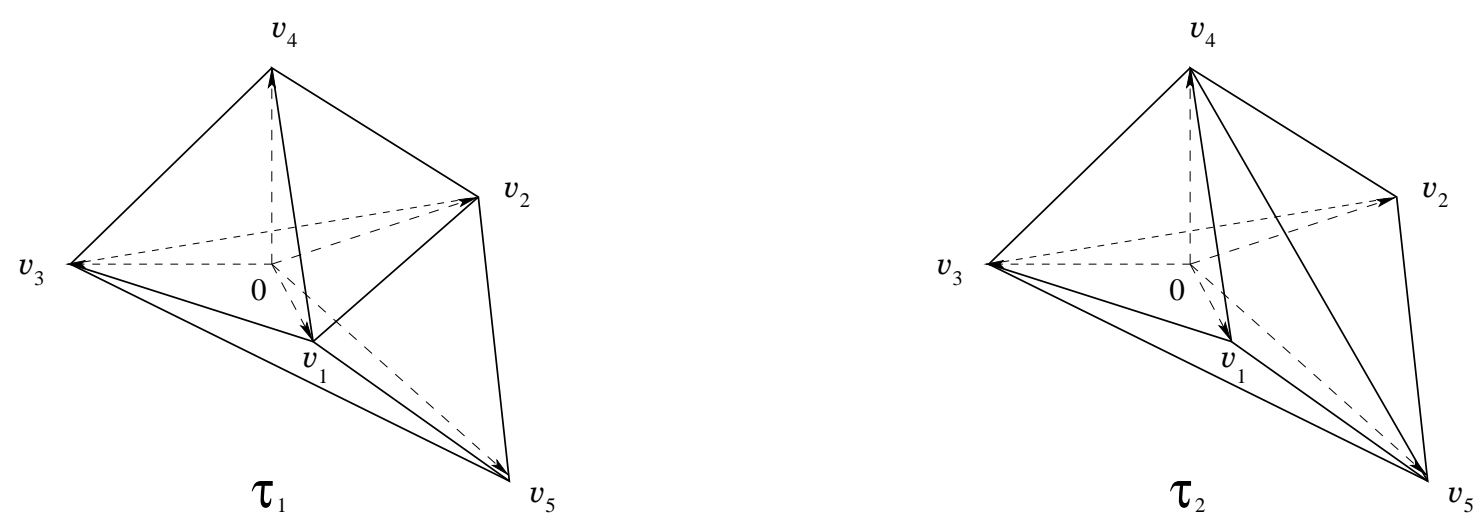

FiguRE 4. Triangulations $\mathcal{T}_{1}$ and $\mathcal{T}_{2}$

defined as $\sigma:=\mathbb{R}_{\geq 0} \tau, \tau \in \mathcal{T}_{1}\left(\tau \in \mathcal{T}_{2}\right)$. Toric varieties $\mathbb{P}_{\Sigma_{1}}$ and $\mathbb{P}_{\Sigma_{2}}$ corresponding to the fans $\Sigma_{1}$ and $\Sigma_{1}$ are related by a flop.

The secondary polytope $\operatorname{Sec}(A)$ is depicted in Figure 5, where the monomials $a_{1}^{4} a_{2}^{4} a_{3}^{4} a_{4}^{3} a_{5}^{3}$ and $a_{1}^{3} a_{2}^{3} a_{3}^{4} a_{4}^{4} a_{5}^{4}$ of $E_{A}(f)$ corresponding to the vertices $v_{\mathcal{T}_{1}}$ and $v_{\mathcal{T}_{2}}$ of $\operatorname{Sec}(A)$ respectively are underlined.

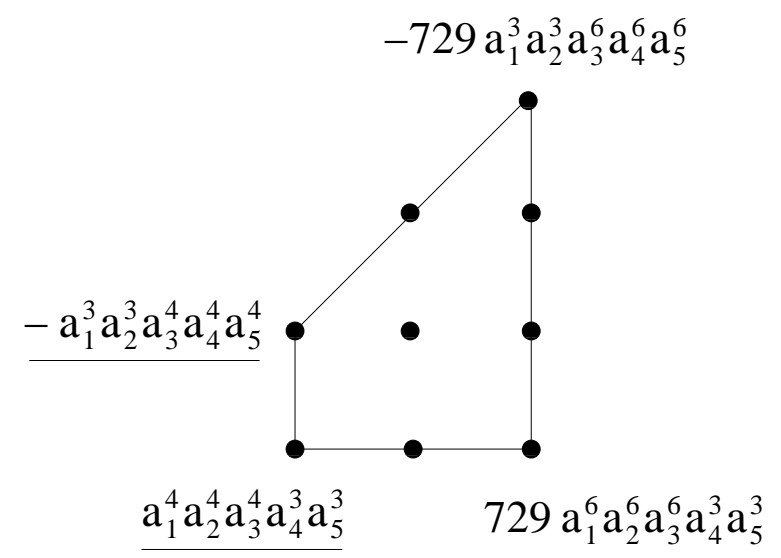

FiguRE 5. Secondary polytope $\operatorname{Sec}(A)$

In oder to find the power series expansion of the toric residue $R_{x_{1} x_{2} x_{4}}\left(a_{1}, \ldots, a_{5}\right)$ at the vertex $v_{\mathcal{T}_{1}}$ of $\operatorname{Sec}(A)$, we rewrite it as follows

$$
R_{x_{1} x_{2} x_{4}}\left(u_{1}, u_{2}\right)=\frac{1-27 u_{1}-81 u_{1} u_{2}}{\left(1-u_{2}\right)\left(1-54 u_{1}+729 u_{1}^{2}-54 u_{1} u_{2}-1458 u_{1}^{2} u_{2}+729 u_{1}^{2} u_{2}^{2}\right)},
$$

where the variables

$$
u_{1}:=a_{1} a_{2} a_{3}, u_{2}:=\frac{a_{4} a_{5}}{a_{1} a_{2}}
$$


correspond to the generators

$$
l_{1}=(1,1,1,0,0), \quad l_{2}=(-1,-1,0,1,1)
$$

of the Mori cone of $\mathbb{P}_{\Sigma_{1}}$. Here are the first terms of the Taylor expansion

$$
R_{x_{1} x_{2} x_{4}}\left(u_{1}, u_{2}\right)=1+27 u_{1}+u_{2}+729 u_{1}^{2}+u_{2}^{2}+19683 u_{1}^{3}+2187 u_{1}^{2} u_{2}+u_{2}^{3}+\cdots
$$

The power series expansion of the toric residue $R_{x_{1} x_{2} x_{4}}\left(a_{1}, \ldots, a_{5}\right)$ at the vertex $v_{\mathcal{T}_{2}} \in \operatorname{Sec}(A)$ (corresponding to the second triangulation $\mathcal{T}_{2}$ ) needs another variables

$$
w_{1}:=u_{1} u_{2}=a_{3} a_{4} a_{5}, w_{2}:=u_{2}^{-1}=\frac{a_{1} a_{2}}{a_{4} a_{5}},
$$

corresponding to the generators

$$
l_{1}^{\prime}=(0,0,1,1,1), l_{2}^{\prime}=(1,1,0,-1,-1)
$$

of the Mori cone of $\mathbb{P}_{\Sigma_{2}}$. We rewrite $R_{x_{1} x_{2} x_{4}}\left(a_{1}, \ldots, a_{5}\right)$ in the form

$$
\begin{aligned}
& R_{x_{1} x_{2} x_{4}}\left(w_{1}, w_{2}\right)= \\
& =\frac{w_{2}-27 w_{1} w_{2}^{2}-81 w_{1} w_{2}}{\left(w_{2}-1\right)\left(1-54 w_{1}-54 w_{1} w_{2}+729 w_{1}^{2}-1458 w_{1}^{2} w_{2}+729 w_{1}^{2} w_{2}^{2}\right)} .
\end{aligned}
$$

We note that the power series expansion of $R_{x_{1} x_{2} x_{4}}\left(u_{1}, u_{2}\right)$ begins with 1 , whereas the series expansion of $R_{x_{1} x_{2} x_{4}}\left(w_{1}, w_{2}\right)$ does not contain a constant term. This fact agrees with different intersection numbers of three divisors $D_{1}, D_{2}, D_{4}$ on $\mathbb{P}_{\Sigma_{1}}$ and $\mathbb{P}_{\Sigma_{2}}$ corresponding to the vectors $v_{1}, v_{2}, v_{4}: \int_{\mathbb{P}_{\Sigma_{1}}}\left[D_{1}\right]\left[D_{2}\right]\left[D_{4}\right]=1$ and $\int_{\mathbb{P}_{\Sigma_{2}}}\left[D_{1}\right]\left[D_{2}\right]\left[D_{4}\right]=0$.

\section{Weighted PROJECTIVE SPACES}

Consider the $d$-dimensional weighted projective space $\mathbb{P}\left(w_{1}, \ldots, w_{n}\right), n=d+1$, where $\operatorname{gcd}\left(w_{1}, \ldots, w_{n}\right)=1$ and

$$
w_{i} \mid\left(w_{1}+\cdots+w_{n}\right), \quad i=1, \ldots, n .
$$

Let $\left\{v_{1}, \ldots, v_{n}\right\} \subset M \cong \mathbb{Z}^{d}$ be the vectors generating $M$ and satisfying the relation

$$
w_{1} v_{1}+\cdots+w_{n} v_{n}=0 .
$$

If we set $A:=\left\{v_{0}=0, v_{1}, \ldots, v_{n}\right\}$, then the polytope $\Delta:=\operatorname{conv}(A) \subset M_{\mathbb{R}}$ is a reflexive simplex. There are exactly two coherent triangulations of $\Delta$ : the triangulation $\mathcal{T}_{1}$ coinciding with the whole polytope $\Delta$, and the triangulation $\mathcal{T}=$ $\mathcal{T}_{2}$ consisting of the union of the $d$-dimensional simplices

$$
\tau_{i}=\operatorname{conv}\left\{0, v_{1}, \ldots, \widehat{v}_{i}, \ldots v_{n}\right\}, \quad i=1, \ldots, n,
$$


where $\widehat{v_{i}}$ means that $v_{i}$ is omitted. Note that $\operatorname{Vol}\left(\tau_{i}\right)=w_{i}$ and $\operatorname{Vol}(\Delta)=\sum_{i=1}^{n} w_{i}$. The fan $\Sigma=\Sigma(\mathcal{T}) \subset M_{\mathbb{R}}$ defining $\mathbb{P}\left(w_{1}, \ldots, w_{n}\right)$ has the generators $\left\{v_{1}, \ldots, v_{n}\right\}$. Let

$$
f(t):=1-\sum_{i=1}^{n} a_{i} t^{v_{i}}
$$

be a generic Laurent polynomial. It is easy to find its principal $A$-determinant from Theorem 4.4.

Proposition 7.1. The secondary polytope $\operatorname{Sec}(A)$ is an interval and the principal A-determinant of $f(t)$ is equal (up to sign) to

$$
E_{A}(f)=\prod_{i=1}^{n} w_{i}^{w_{i}} a_{i}^{w_{1}+\cdots+\widehat{w}_{i}+\cdots+w_{n}}-\left(\sum_{i=1}^{n} w_{i}\right)^{\sum_{i=1}^{n} w_{i}}\left(a_{1} \cdots a_{n}\right)^{\sum_{i=1}^{n} w_{i}}
$$

where $\widehat{w}_{i}$ means that $w_{i}$ is omitted. The first summand in $E_{A}(f)$ corresponds to the triangulation $\mathcal{T}$.

Theorem 7.2. Let $P\left(x_{1}, \ldots, x_{n}\right) \in \mathbb{Q}\left[x_{1}, \ldots, x_{n}\right]$ be an arbitrary homogeneous polynomial of degree $d$. Denote $y:=a_{1}^{w_{1}} \cdots a_{n}^{w_{n}}$. Then the toric residue

$$
R_{P}(a)=(-1)^{d} \operatorname{Res}_{f}\left(t_{0}^{d} P\left(a_{1} t^{v_{1}}, \ldots, a_{n} t^{v_{n}}\right)\right)
$$

is equal to the rational function

$$
R_{P}\left(a_{1}^{w_{1}} \cdots a_{n}^{w_{n}}\right)=R_{P}(y)=\frac{\nu \cdot P\left(w_{1}, \ldots, w_{n}\right)}{1-\mu y},
$$

where

$$
\nu:=\frac{1}{w_{1} \cdots w_{n}}, \quad \mu:=\left(\frac{\left(\sum_{i=1}^{n} w_{i}\right)^{\sum_{i=1}^{n} w_{i}}}{\prod_{i=1}^{n} w_{i}^{w_{i}}}\right) .
$$

Proof. By Theorem 2.9 $(i)$, the toric residue $R_{P}$ can be computed as a sum over the critical points $\xi$ of $f$ :

$$
R_{P}=(-1)^{d} \sum_{\xi \in V_{f}} \frac{P\left(a_{1} \xi^{v_{1}}, \ldots, a_{n} \xi^{v_{n}}\right)}{f(\xi) H_{f}^{0}(\xi)} .
$$

If $\xi$ is a critical point of $f$, i.e., a solution of the system of equations

$$
t_{1} \frac{\partial f}{\partial t_{1}}(t)=\cdots=t_{d} \frac{\partial f}{\partial t_{d}}(t)=0, \quad t \in\left(\mathbb{C}^{*}\right)^{d},
$$


then

$$
a_{1} \frac{\xi^{v_{1}}}{w_{1}}=\cdots=a_{n} \frac{\xi^{v_{n}}}{w_{n}}=z
$$

and

$$
z^{w_{1}+\cdots+w_{n}}=\left(\frac{a_{1}}{w_{1}}\right)^{w_{1}} \cdots\left(\frac{a_{n}}{w_{n}}\right)^{w_{n}} .
$$

These relations simplify our computations. For example, we may write at the critical points of $f$ :

$$
f(\xi)=1-\left(\sum_{i=1}^{n} w_{i}\right) z
$$

and

$$
P\left(a_{1} \xi^{v_{1}}, \ldots, a_{n} \xi^{v_{n}}\right)=P\left(w_{1}, \ldots, w_{n}\right) z^{d} .
$$

The value of the polynomial

$$
H_{f}^{0}(t)=\operatorname{det}\left(\left(t_{i} \frac{\partial}{\partial t_{i}}\right)\left(t_{j} \frac{\partial}{\partial t_{j}}\right) f\right)_{1 \leq i, j \leq d}
$$

at a critical point $\xi$ of $f$ equals

$$
H_{f}^{0}(\xi)=(-1)^{d} w_{1} \cdots w_{n}\left(\sum_{i=1}^{n} w_{i}\right) z^{d} .
$$

Since the summation over the critical points is equivalent to the summation over the roots of the equation (7), we get

$$
\begin{aligned}
& R_{P}(y)=\sum_{z^{w_{1}+\cdots+w_{n}}=\left(\frac{a_{1}}{w_{1}}\right)^{w_{1}} \ldots\left(\frac{a_{n}}{w_{n}}\right)^{w_{n}}} \frac{P\left(w_{1}, \ldots, w_{n}\right)}{w_{1} \cdots w_{n}\left(\sum_{i=1}^{n} w_{i}\right)\left(1-\left(\sum_{i=1}^{n} w_{i}\right) z\right)} \\
& =\frac{P\left(w_{1}, \ldots, w_{n}\right)}{w_{1} \ldots w_{n}} \sum_{b \geq 0}\left(\sum_{i=1}^{n} w_{i}\right)^{\left(\sum_{i=1}^{n} w_{i}\right) b}\left(\frac{a_{1}^{w_{1}} \cdots a_{n}^{w_{n}}}{w_{1}^{w_{1}} \cdots w_{n}^{w_{n}}}\right)^{b} \\
& =\frac{\nu \cdot P\left(w_{1}, \ldots, w_{n}\right)}{1-\mu y}
\end{aligned}
$$

as required.

Now we show that the expansion of $R_{P}(y)$ at the vertex $v_{\mathcal{T}} \in \operatorname{Sec}(A)$ corresponding to the triangulation $\mathcal{T}$ coincides with the generating function of intersection numbers: 
Theorem 7.3. Let $P\left(x_{1}, \ldots, x_{n}\right)$ be any homogeneous polynomial in $\mathbb{Q}\left[x_{1}, \ldots, x_{n}\right]$ of degree $d$. The generating function of intersection numbers on the MorrisonPlesser moduli spaces has the form

$$
I_{P}(y)=\nu \cdot P\left(w_{1}, \ldots, w_{n}\right) \sum_{b \geq 0} \mu^{b} y^{b}=\frac{\nu \cdot P\left(w_{1}, \ldots, w_{n}\right)}{1-\mu y} .
$$

Proof. The Morrison-Plesser moduli spaces $\mathbb{P}_{\beta}$ for the weighted projective space $\mathbb{P}=\mathbb{P}\left(w_{1}, \ldots, w_{n}\right)$ are also weighted projective spaces of dimension $\left(\sum_{i=1}^{n} w_{i}\right) b+d$. Let $D_{1}, \ldots, D_{n}$ be the divisors corresponding to the vectors $v_{1}, \ldots, v_{n}$. We have the following relations between the torus-invariant divisors on $\mathbb{P}_{\beta}$ modulo rational equivalence:

$$
\frac{\left[D_{1}\right]}{w_{1}}=\cdots=\frac{\left[D_{n}\right]}{w_{n}}=\left[D_{0}\right]
$$

Then the Mori cone $K_{\text {eff }}(\mathbb{P})$ consists of $\beta=(b), b \geq 0$. The Morrison-Plesser class is exactly

$$
\Phi_{\beta}=\left(\left(\sum_{i=1}^{n} w_{i}\right)\left[D_{0}\right]\right)^{\left(\sum_{i=1}^{n} w_{i}\right) b}
$$

and the generating function of intersection numbers on $\mathbb{P}_{\beta}$ can be written as

$$
I_{P}(y)=\sum_{b \geq 0}\left\langle P\left(\left[D_{1}\right], \ldots,\left[D_{n}\right]\right)\left(\left(\sum_{i=1}^{n} w_{i}\right)\left[D_{0}\right]\right)^{\left(\sum_{i=1}^{n} w_{i}\right) b}\right\rangle_{\beta} y^{b} .
$$

Using $\left\langle\left[D_{0}\right]^{\left(\sum_{i=1}^{n} w_{i}\right) b+d}\right\rangle_{\beta}=1 /\left(w_{1}^{w_{1} b+1} \cdots w_{n}^{w_{n} b+1}\right)$, we obtain

$$
\begin{aligned}
I_{P}(y) & =P\left(w_{1}, \ldots, w_{n}\right) \sum_{b \geq 0}\left(\sum_{i=1}^{n} w_{i}\right)^{\left(\sum_{i=1}^{n} w_{i}\right) b}\left\langle\left[D_{0}\right]^{\left.\sum_{i=1}^{n} w_{i}\right) b+d}\right\rangle_{\beta} y^{b} \\
& =P\left(w_{1}, \ldots, w_{n}\right) \sum_{b \geq 0}\left(\sum_{i=1}^{n} w_{i}\right)^{\left(\sum_{i=1}^{n} w_{i}\right) b} \frac{1}{\frac{w_{1}^{w_{1} b+1} \cdots w_{n}^{w_{n} b+1}}{y^{b}}} y^{b} \\
& =\nu \cdot P\left(w_{1}, \ldots, w_{n}\right) \sum_{b \geq 0} \mu^{b} y^{b}=\frac{\nu \cdot P\left(w_{1}, \ldots, w_{n}\right)}{1-\mu y} .
\end{aligned}
$$




\section{Product of projective spaces}

In this section we check the Toric Residue Mirror Conjecture in the case $\mathbb{P}=$ $\mathbb{P}^{d_{1}} \times \cdots \times \mathbb{P}^{d_{r}}$.

For all $j=1, \ldots, r$, we set $n_{j}:=d_{j}+1$ and denote by $M_{j}$ the free abelian group of rank $d_{j}$ generated by the elements $v_{j 1}, \ldots, v_{j n_{j}}$ satisfying the linear relation:

$$
v_{j 1}+\cdots+v_{j n_{j}}=0 .
$$

Let $M:=M_{1} \oplus \cdots \oplus M_{r}$. Consider a Laurent polynomial

$$
f(t)=1-\sum_{i_{1}=1}^{n_{1}} a_{1 i_{1}} t^{v_{1 i_{1}}}-\cdots-\sum_{i_{r}=1}^{n_{r}} a_{r i_{r}} t^{v_{r i}}
$$

with support in the reflexive polytope

$$
\Delta=\operatorname{conv}\left(\left\{v_{11}, \ldots, v_{1 n_{1}}, \ldots, v_{r 1}, \ldots, v_{r n_{r}}\right\}\right) \subset M_{\mathbb{R}} .
$$

The fan $\Sigma \subset M_{\mathbb{R}}$ consisting of cones over all faces of $\Delta$ determines the toric variety $\mathbb{P}=\mathbb{P}^{d_{1}} \times \cdots \times \mathbb{P}^{d_{r}}$ of dimension $d:=d_{1}+\cdots+d_{r}$. Consider $n:=n_{1}+\cdots+n_{r}$ variables $x_{j i}\left(1 \leq i \leq n_{j}, 1 \leq j \leq r\right)$. We will use the following notations:

$$
\begin{gathered}
x^{k}:=x_{11}^{k_{11}} \cdots x_{1 n_{1}}^{k_{1 n_{1}}} \cdots x_{r 1}^{k_{r 1}} \cdots x_{r n_{r}}^{k_{r n_{r}}}, \\
k_{j}:=k_{j 1}+\cdots+k_{j n_{j}}, \quad u_{j}:=n_{j}^{n_{j}} a_{j 1} \cdots a_{j n_{j}}, \quad j=1, \ldots, r .
\end{gathered}
$$

Let $K=\mathbb{C}\left(u_{1}, \ldots, u_{r}\right)$ be the field of rational functions in $u_{1}, \ldots, u_{r}$. Let $z_{j}$ be a root of the equation $z_{j}^{n_{j}}=u_{j}(1 \leq j \leq r)$. We obtain a finite Galois extension $L=\mathbb{C}\left(z_{1}, \ldots, z_{r}\right)$ of $K$ of degree $[L: K]=n_{1} \cdots n_{r}$. One has the algebraic trace map

$$
\operatorname{tr}_{L / K}: L \rightarrow K,
$$

which can be defined by the formula

$$
\operatorname{tr}_{L / K}(g)=\sum_{\substack{z_{1}^{n_{1}}=u_{1} \\ z_{r}^{n_{r}}=u_{r}}} g\left(z_{1}, \ldots, z_{r}\right),
$$

where the sum runs over all roots of the system of equations $z_{j}^{n_{j}}=u_{j}(1 \leq j \leq r)$.

Theorem 8.1. Let $P(x)=x^{k}$ be any monomial of degree d (i.e., $\sum_{j=1}^{r} k_{j}=d$ ). Then the toric residue $R_{P}$ corresponding to the monomial $x^{k}$ is the rational function in $u_{1}, \ldots, u_{r}$ :

$$
R_{x^{k}}(u)=n_{1}^{d_{1}-k_{1}-1} \cdots n_{r}^{d_{r}-k_{r}-1} \operatorname{tr}_{L / K}\left(\frac{1}{z_{1}^{d_{1}-k_{1}} \cdots z_{r}^{d_{r}-k_{r}}\left(1-z_{1}-\cdots-z_{r}\right)}\right) .
$$


Proof. The proof is based on the formula from Theorem 2.9 $(i)$ which express the toric residue $R_{P}$ as the following sum over the critical points $\xi$ of $f$ :

$$
R_{P}=(-1)^{d} \sum_{\xi \in V_{f}} \frac{P\left(a_{11} \xi^{v_{11}}, \ldots, a_{r n_{r}} \xi^{v_{r n_{r}}}\right)}{f(\xi) H_{f}^{0}(\xi)} .
$$

If $\xi$ is a critical point of $f$, then the vanishing of partial derivatives of $f$ implies:

$$
a_{j 1} \xi^{v_{j 1}}=\cdots=a_{j n_{j}} \xi^{v_{j n_{j}}}, \quad j=1, \ldots, r .
$$

We set $z_{j}:=a_{j 1} \xi^{v_{j 1}}=\cdots=a_{j n_{j}} \xi^{v_{j n_{j}}}$. It follows from $v_{j 1}+\cdots+v_{j n_{j}}=0$ that

$$
z_{j}^{n_{j}}=a_{j 1} \cdots a_{j n_{j}}, \quad j=1, \ldots, r .
$$

Denote the product $a_{j 1} \cdots a_{j n_{j}}$ by $y_{j}$. It is easy to compute the values of the polynomials $f$ and $H_{f}^{0}(t)$ at a critical point $\xi$ :

$$
\begin{gathered}
f(\xi)=1-n_{1} z_{1}-\cdots-n_{r} z_{r}, \\
H_{f}^{0}(\xi)=(-1)^{d} n_{1} \cdots n_{r} z_{1}^{d_{1}} \cdots z_{r}^{d_{r}} .
\end{gathered}
$$

On the other hand, we have

$$
P\left(a_{11} \xi^{v_{11}}, \ldots, a_{r n_{r}} \xi^{v_{r n_{r}}}\right)=z_{1}^{k_{1}} \cdots z_{r}^{k_{r}} .
$$

Thus, we obtain

$$
R_{x^{k}}(u)=\frac{1}{n_{1} \cdots n_{r}} \sum_{\substack{z_{1}^{n_{1}}=y_{1} \\ z_{r}^{n_{r}}=y_{r}}} \frac{z_{1}^{k_{1}} \cdots z_{r}^{k_{r}}}{\left(1-n_{1} z_{1}-\cdots-n_{r} z_{r}\right) z_{1}^{d_{1}} \cdots z_{r}^{d_{r}}} .
$$

Setting $u_{j}:=n_{j}^{n_{j}} y_{j}(1 \leq j \leq r)$, we get the required formula.

In order to compute the generating functions of intersection numbers $I(P, \beta)$ on the Morrison-Plesser moduli spaces associated with $\mathbb{P}$, we remark that the Mori cone $K_{\text {eff }}(\mathbb{P})$ is a simplicial $r$-dimensional cone generated by the canonical basis of $H_{2}(\mathbb{P}, \mathbb{Z}) \cong \mathbb{Z}^{r}$. Moreover, all Morrison-Plesser moduli spaces $\mathbb{P}_{\beta}$ are projective spaces $\mathbb{P}^{n_{1} b_{1}+d_{1}} \times \cdots \times \mathbb{P}^{n_{r} b_{r}+d_{r}}$, where $\beta$ can be identified with a lattice point $\left(b_{1}, \ldots, b_{r}\right) \in K_{\text {eff }}(\mathbb{P})=\mathbb{R}_{\geq 0}^{r}$. Therefore, the generating function

$$
I_{P}(y)=\sum_{\beta \in K_{\mathrm{eff}}(\mathbb{P})} I(P, \beta) a^{\beta},
$$

can be rewritten as

$$
I_{P}(y)=\sum_{b_{1}, \ldots, b_{r} \geq 0} I(P, \beta) y_{1}^{b_{1}} \cdots y_{r}^{b_{r}}
$$

where $y_{j}=a_{j 1} \cdots a_{j n_{j}}(1 \leq j \leq r)$. 
Theorem 8.2. Let $u_{j}=n_{j}^{n_{j}} y_{j}(1 \leq j \leq r)$. Then the generating function of intersection numbers $I\left(x^{k}, \beta\right)$ can be written as

$$
I_{x^{k}}(u)=n_{1}^{d_{1}-k_{1}} \cdots n_{r}^{d_{r}-k_{r}} \sum_{b_{1}, \ldots, b_{r} \geq 0} \frac{\left(n_{1} b_{1}+\cdots+n_{r} b_{r}\right) !}{\left(n_{1} b_{1}+d_{1}-k_{1}\right) ! \cdots\left(n_{r} b_{r}+d_{r}-k_{r}\right) !} u_{1}^{b_{1}} \cdots u_{r}^{b_{r}} .
$$

Proof. Let $\left[H_{j}\right]$ be the hyperplane class on $\mathbb{P}^{d_{j}}$. Since $\mathbb{P}$ contains exactly $n_{j}$ torusinvariant divisors having the class $\left[H_{j}\right]$, the intersection number $I\left(x^{k}, \beta\right)$ equals

$$
\left\langle\left[H_{1}\right]^{k_{1}} \cdots\left[H_{r}\right]^{k_{r}}\left(n_{1}\left[H_{1}\right]+\cdots+n_{r}\left[H_{r}\right]\right)^{n_{1} b_{1}+\cdots+n_{r} b_{r}}\right\rangle_{\beta} .
$$

We have

$$
I\left(x^{k}, \beta\right)=\sum_{m_{1}+\cdots+m_{r}=m} \frac{m !}{m_{1} ! \cdots m_{r} !} n_{1}^{m_{1}} \cdots n_{r}^{m_{r}}\left\langle\left[H_{1}\right]^{m_{1}+k_{1}} \cdots\left[H_{r}\right]^{m_{r}+k_{r}}\right\rangle_{\beta},
$$

where $m=\sum_{j=1}^{r} n_{j} b_{j}$. The intersection theory on $\mathbb{P}_{\beta}$ implies

$$
\left\langle\left[H_{1}\right]^{l_{1}} \cdots\left[H_{r}\right]^{l_{r}}\right\rangle_{\beta}= \begin{cases}1, & l_{j}=n_{j} b_{j}+d_{j}, \quad j=1, \ldots, r \\ 0, & \text { otherwise }\end{cases}
$$

So we obtain

$$
I_{x^{k}}(u)=n_{1}^{d_{1}-k_{1}} \cdots n_{r}^{d_{r}-k_{r}} \sum_{b_{1}, \ldots, b_{r} \geq 0} \frac{\left(n_{1} b_{1}+\cdots+n_{r} b_{r}\right) !}{\left(n_{1} b_{1}+d_{1}-k_{1}\right) ! \cdots\left(n_{r} b_{r}+d_{r}-k_{r}\right) !} u_{1}^{b_{1}} \cdots u_{r}^{b_{r}},
$$

where $u_{j}=n_{j}^{n_{j}} y_{j}(1 \leq j \leq r)$.

It is sufficient to verify the Toric Residue Mirror Conjecture (Conjecture 4.6) for any monomial $P=x^{k}$ of degree $d$. By Theorem 8.1 and Theorem 8.2, the equality $R_{x^{k}}(u)=I_{x^{k}}(u)$ follows from

Proposition 8.3. Let

$$
T_{k}(u):=\operatorname{tr}_{L / K}\left(\frac{1}{z_{1}^{d_{1}-k_{1}} \cdots z_{r}^{d_{r}-k_{r}}\left(1-z_{1}-\cdots-z_{r}\right)}\right),
$$

then $T_{k}$ has the following power series expansion

$$
T_{k}(u)=n_{1} \cdots n_{r} \sum_{b_{1}, \ldots, b_{r} \geq 0} \frac{\left(n_{1} b_{1}+\cdots+n_{r} b_{r}\right) !}{\left(n_{1} b_{1}+d_{1}-k_{1}\right) ! \cdots\left(n_{r} b_{r}+d_{r}-k_{r}\right) !} u_{1}^{b_{1}} \cdots u_{r}^{b_{r}}
$$


Proof. We have the chain of equalities:

$$
\begin{aligned}
& T_{k}(u)=\sum_{\substack{z_{1}^{n_{1}}=u_{1} \\
\cdots \\
z_{r}^{n_{r}}=u_{r}}} \frac{1}{z_{1}^{d_{1}-k_{1}} \cdots z_{r}^{d_{r}-k_{r}}} \sum_{b_{1}, \ldots, b_{r} \geq 0} \frac{\left(b_{1}+\cdots+b_{r}\right) !}{b_{1} ! \cdots b_{r} !} z_{1}^{b_{1}} \cdots z_{r}^{b_{r}} \\
= & \sum_{\substack{z_{1}^{n_{1}}=u_{1} \\
\cdots \\
z_{r}^{n_{r}}=u_{r}}} \frac{1}{z_{1}^{d_{1}-k_{1}} \cdots z_{r}^{d_{r}-k_{r}}} \sum_{b} \frac{\left(b_{1}+\cdots+b_{r}-\sum_{i=1}^{r}\left(d_{i}-k_{i}\right)\right) !}{b_{1} ! \cdots b_{r} !} z_{1}^{b_{1}} \cdots z_{r}^{b_{r}} \\
= & \sum_{\substack{z_{1}^{n_{1}}=u_{1} \\
\cdots z_{r}^{n_{r}}=u_{r}}} \frac{1}{z_{1}^{d_{1}-k_{1}} \cdots z_{r}^{d_{r}-k_{r}}} \sum_{b} \frac{\left(b_{1}+\cdots+b_{r}\right) !}{\left(b_{1}+d_{1}-k_{1}\right) ! \cdots\left(b_{r}+d_{r}-k_{r}\right) !} z_{1}^{b_{1}+d_{1}-k_{1}} \cdots z_{r}^{b_{r}+d_{r}-k_{r}} \\
= & \sum_{\substack{z_{1}^{n_{1}}=u_{1} \\
z_{r}^{n_{r}}=u_{r}}} \sum_{b} \frac{\left(b_{1}+\cdots+b_{r}\right) !}{\left(b_{1}+d_{1}-k_{1}\right) ! \cdots\left(b_{r}+d_{r}-k_{r}\right) !} z_{1}^{b_{1}} \cdots z_{r}^{b_{r}} \\
= & n_{1} \cdots n_{r} \sum_{b_{1}, \ldots, b_{r} \geq 0} \frac{\left(n_{1} b_{1}+\cdots+n_{r} b_{r}\right) !}{\left(n_{1} b_{1}+d_{1}-k_{1}\right) ! \cdots\left(n_{r} b_{r}+d_{r}-k_{r}\right) !} u_{1}^{b_{1}} \cdots u_{r}^{b_{r}},
\end{aligned}
$$

where in the last row we have used the identity

$$
\sum_{z_{j}^{n_{j}}=u_{j}} z_{j}^{b_{j}}= \begin{cases}n_{j} u_{j}^{b_{j}}, & b_{j}=k n_{j}, \\ 0, & b_{j} \neq k n_{j}, \quad k=1,2, \ldots\end{cases}
$$

for each $j=1, \ldots, r$.

\section{Yukawa $(d-1)$-Point functions for Calabi-Yau hypersurfaces}

Let $\Delta \subset M_{\mathbb{R}}$ be a reflexive polytope of dimension $d, A=\left\{0, v_{1}, \ldots, v_{n}\right\}$ a finite subset in $\Delta \cap M$ containing 0 and all vertices of $\Delta, f(t)$ a Laurent polynomial in the variables $t_{1}, \ldots, t_{d}$ with support in $\Delta$ of the form

$$
f(t):=1-\sum_{i=1}^{n} a_{i} t^{v_{i}}
$$

We denote by $Z_{f}$ a Calabi-Yau hypersurface defined by the equation $f=0$ in the torus $\mathbb{T} \cong\left(\mathbb{C}^{*}\right)^{d}$. Let

$$
\Omega:=\operatorname{Res}\left(\frac{1}{f} \frac{d t_{1}}{t_{1}} \wedge \cdots \wedge \frac{d t_{d}}{t_{d}}\right)
$$


be the image of the canonical $d$-form

$$
\frac{1}{f} \frac{d t_{1}}{t_{1}} \wedge \cdots \wedge \frac{d t_{d}}{t_{d}} \in H^{d}\left(\mathbb{T} \backslash Z_{f}\right)
$$

under the Poincaré residue mapping

$$
\text { Res : } H^{d}\left(\mathbb{T} \backslash Z_{f}\right) \rightarrow H^{d-1}\left(Z_{f}\right)
$$

Definition 9.1. Assign to each nonzero lattice point $v_{i} \in A$ a variable $x_{i}$. Let $Q\left(x_{1}, \ldots, x_{n}\right) \in \mathbb{Q}\left[x_{1}, \ldots, x_{n}\right]$ be a homogeneous polynomial of degree $d-1$. The $Q$-Yukawa $(d-1)$-point function is defined by the formula

$$
Y_{Q}\left(a_{1}, \ldots, a_{n}\right):=(-1)^{\frac{(d-1)(d-2)}{2}} \frac{1}{(2 \pi i)^{d-1}} \int_{Z_{f}} \Omega \wedge Q\left(a_{1} \frac{\partial}{\partial a_{1}}, \ldots, a_{n} \frac{\partial}{\partial a_{n}}\right) \Omega
$$

where the differential operators $a_{1} \partial / \partial a_{1}, \ldots, a_{n} \partial / \partial a_{n}$ are determined by the GaußManin connection.

Remark 9.2. The sign $(-1)^{\frac{(d-1)(d-2)}{2}}$ in the definition of the Yukawa $(d-1)$-point function is inherited by the variation of the Hodge structure (see in [CK, Section 8.6.3]). The 3-point Yukawa functions are also called Yukawa couplings.

Example 9.3. Consider the mirror family of Calabi-Yau hypersurfaces in the projective space $\mathbb{P}^{d}$ defined by the Laurent polynomial

$$
f(t)=1-\sum_{i=1}^{n} a_{i} t^{v_{i}}, \quad n=d+1
$$

where $v_{1}, \ldots, v_{d}$ form a basis of the lattice $M$ and

$$
v_{d+1}=-\left(v_{1}+\cdots+v_{d}\right) .
$$

If we set $y:=a_{1} \cdots a_{n}$, then the $Q$-Yukawa $(d-1)$-point function is equal to

$$
Y_{Q}(y)=\frac{n Q(1, \ldots, 1)}{1-n^{n} y}, \quad \operatorname{deg} Q=d-1
$$

(see e.g. $\mathrm{JN}$ ). In the particular case $d=4$ and $Q(x)=x^{3}$ the formula (9) gives the Yukawa 3-point function for mirrors of Calabi-Yau quintic hypersurfaces in $\mathbb{P}^{4}$

$$
Y_{x^{3}}(y)=\frac{5}{1-5^{5} y}
$$

which is well-known from COGP.

In order to establish the relation between Yukawa $(d-1)$-point functions and toric residues, we need the notion of homogeneous coordinate ring of a toric variety [Cox1]. Let $\Sigma$ be a complete simplicial fan in $N_{\mathbb{R}}$ defining a projective simplicial toric variety $\mathbb{P}=\mathbb{P}_{\Sigma}, \Sigma(1):=\left\{e_{1}, \ldots, e_{r}\right\}$ the set of generators of 1-dimensional 
cones in $\Sigma$, and $z_{1}, \ldots, z_{r}$ the corresponding homogeneous coordinates. The polynomial ring

$$
S(\mathbb{P}):=\mathbb{C}\left[z_{1}, \ldots, z_{r}\right]
$$

having a natural grading by $\mathrm{Cl}(\mathbb{P})$ is called the homogeneous coordinate ring of $\mathbb{P}$.

Let $H=\sum_{i=1}^{r} c_{i} D_{i}$ be a big and nef divisor on $\mathbb{P}$. One obtains the convex polytope $\Delta_{H} \subset M_{\mathbb{R}}$ as intersection of $r$ half-spaces $\left\langle m, e_{i}\right\rangle \geq-c_{i}(1 \leq i \leq r)$. For any lattice point $m$ in $k \Delta_{H}$, one has $\left\langle m, e_{i}\right\rangle+k c_{i} \geq 0(1 \leq i \leq r)$. Thus we can define a mapping $S_{\Delta_{H}} \rightarrow S(\mathbb{P})$ which sends a monomial $t_{0}^{k} t^{m} \in S_{\Delta_{H}}^{k}$ to the monomial $\prod_{i=1}^{r} z_{i}^{\left\langle m, e_{i}\right\rangle+k c_{i}} \in S(\mathbb{P})$. This mapping yields an isomorphism of graded rings

$$
S_{\Delta_{H}} \cong \bigoplus_{k=0}^{\infty} S(\mathbb{P})_{k \alpha},
$$

where $\alpha$ is the class of $H$ in $\mathrm{Cl}(\mathbb{P})$. For all $k$, this isomorphism identifies the subspace $I_{\Delta_{H}}^{k} \subset S_{\Delta_{H}}^{k}$ with the image of $S(\mathbb{P})_{k \alpha-\omega_{0}}$ in $S(\mathbb{P})_{k \alpha}$ under the mapping

$$
S(\mathbb{P})_{k \alpha-\omega_{0}} \stackrel{\prod_{i=1}^{r} z_{i}}{\longrightarrow} S(\mathbb{P})_{k \alpha}
$$

where $\omega_{0} \in \mathrm{Cl}(\mathbb{P})$ is the anticanonical class of $\mathbb{P}$. This bijection allows to compare our notion of toric residue from Section 2 with the definition of toric residue given by D. Cox in Cox2.

By the isomorphism (10), we identify the regular sequence $G=\left(G_{0}, \ldots, G_{d}\right)$ of elements in $S_{\Delta_{H}}^{1}$ with its image in $S(\mathbb{P})_{\alpha}$. Thus, by Cox2, Theorem 5.1] (see also [Mav1, Thorem 4.8], where the theorem of Cox was extended to the case when $H$ is big and nef), the toric residue mapping (2) coincides with the residue mapping

$$
\operatorname{Res}_{G}: S(\mathbb{P})_{\rho} \rightarrow \mathbb{C}, \quad \rho=(d+1) \alpha-\omega_{0}
$$

considered by Cox and induces the canonical isomorphism

$$
S(\mathbb{P})_{\rho} /\left\langle G_{0}, \ldots, G_{d}\right\rangle_{\rho} \cong \mathbb{C}
$$

Remark 9.4. In particular, let $\alpha=\omega_{0}$, i.e., $\Delta_{H}$ be a reflexive polytope (we denote it shortly by $\Delta), f(t)$ be a generic Laurent polynomial with support $\Delta$ defining a Calabi-Yau hypersurface $Z_{f} \subset \mathbb{T}$, and $F_{0}(z), \ldots, F_{d}(z) \in S(\mathbb{P})_{\omega_{0}}$ are the images of the following regular sequence in $S_{\Delta}^{1}$ :

$$
t_{0} f, t_{0} t_{1} \partial f / \partial t_{1}, \ldots, t_{0} t_{d} \partial f / \partial t_{d} .
$$

Then the isomorphism (4) from Section 2 coincides with

$$
S(\mathbb{P})_{\rho} /\left\langle F_{0}, \ldots, F_{d}\right\rangle_{\rho} \cong \mathbb{C}, \quad \rho=d \omega_{0} .
$$


Definition 9.5 (Mav1]). Given a subset $I=\left\{e_{i_{0}}, \ldots, e_{i_{d}}\right\} \subset \Sigma(1)$ consisting of $d+1$ elements and an integral basis $m_{1}, \ldots, m_{d}$ of the lattice $M$, denote by $c_{I}$ the determinant of the $(d+1) \times(d+1)$-matrix obtained from the matrix $\left(\left\langle m_{j}, e_{i_{k}}\right\rangle_{1 \leq j \leq d, i_{k} \in I}\right)$ by adding the first row $(1, \ldots, 1)$.

The following statement shows how Yukawa $(d-1)$-point functions can be computed by means of toric residues $\operatorname{Res}_{F_{I}}$ with respect to some sequence $F_{I}$ of polynomials in the homogeneous coordinate ring of a toric variety (it is a reformulation of the result in [Mav1, p. 104]):

Theorem 9.6. Let $\Delta$ be a d-dimensional reflexive polytope and $F_{0}(z) \in S(\mathbb{P})_{\omega_{0}} a$ generic homogeneous polynomial as in Remark 9.4. Choose a subset $I=\left\{e_{i_{0}}, \ldots, e_{i_{d}}\right\} \subset$ $\Sigma(1)$ is such that $c_{I} \neq 0$ (see Definition 9.5) and define the sequence $F_{I}$ of homogeneous polynomials as $F_{I}=\left(z_{i_{0}} \partial F_{0} / \partial z_{i_{0}}, \ldots, z_{i_{d}} \partial F_{0} / \partial z_{i_{d}}\right)$. Let $Q\left(x_{1}, \ldots, x_{n}\right) \in$ $\mathbb{Q}\left[x_{1}, \ldots, x_{n}\right]$ be a homogeneous polynomial of degree $d-1$. We set

$$
q(t):=(-1)^{d-1} t_{0}^{d-1} Q\left(a_{1} t^{v_{1}}, \ldots, a_{n} t^{v_{n}}\right)
$$

and denote by $\tilde{q}(z) \in S(\mathbb{P})_{(d-1) \omega_{0}}$ the image of the polynomial $q(t)$ under the isomorphism (10). Then the $Q$-Yukawa $(d-1)$-point function is equal to the toric residue

$$
Y_{Q}(a)=-c_{I} \operatorname{Res}_{F_{I}}\left(\tilde{q}(z) \cdot \prod_{i=1}^{r} z_{i}\right) .
$$

There exists another formula for Yukawa $(d-1)$-point function $Y_{Q}(a)$ which does not depend on the choice of a subset $I=\left\{e_{i_{0}}, \ldots, e_{i_{d}}\right\} \subset \Sigma(1)$ :

Theorem 9.7. Let $Q\left(x_{1}, \ldots, x_{n}\right) \in \mathbb{Q}\left[x_{1}, \ldots, x_{n}\right]$ be a homogeneous polynomial of degree $(d-1)$ and

$$
P\left(x_{1}, \ldots, x_{n}\right):=\left(x_{1}+\cdots+x_{n}\right) Q\left(x_{1}, \ldots, x_{n}\right) .
$$

Then the $Q$-Yukawa $(d-1)$-point function is equal to the toric residue

$$
Y_{Q}\left(a_{1}, \ldots, a_{n}\right)=(-1)^{d} \operatorname{Res}_{f}\left(t_{0}^{d} P\left(a_{1} t^{v_{1}}, \ldots, a_{n} t^{v_{n}}\right)\right) .
$$

Proof. It follows from the definition of toric residue that

$$
\begin{aligned}
\operatorname{Res}_{f}\left(t_{0}^{d} P\left(a_{1} t^{v_{1}}, \ldots, a_{n} t^{v_{n}}\right)\right) & =\operatorname{Res}_{f}\left(t_{0}^{d}(1-f(t)) Q\left(a_{1} t^{v_{1}}, \ldots, a_{n} t^{v_{n}}\right)\right) \\
& =\operatorname{Res}_{f}\left(t_{0}^{d} Q\left(a_{1} t^{v_{1}}, \ldots, a_{n} t^{v_{n}}\right)\right) .
\end{aligned}
$$

Let $F_{0}(z), \ldots, F_{d}(z) \in S(\mathbb{P})_{\omega_{0}}$ be the homogeneous polynomials as in Remark 9.4 . Then the last residue can be written in homogeneous coordinates as

$$
\operatorname{Res}_{f}\left(t_{0}^{d} Q\left(a_{1} t^{v_{1}}, \ldots, a_{n} t^{v_{n}}\right)\right)=(-1)^{d-1} \operatorname{Res}_{F}\left(\tilde{q}(z) \cdot \prod_{i=1}^{r} z_{i}\right)
$$


where $\operatorname{Res}_{F}$ is the Cox's residue with respect to the sequence $F=\left(F_{0}, \ldots, F_{d}\right)$. Compare this residue with the residue from Theorem 9.6. To do this, note that the reflexivity of $\Delta$ implies that each monomial $t_{0} t^{m} \in S_{\Delta}^{1}$ maps to the monomial $\prod_{i=1}^{r} z_{i}^{\left\langle m, e_{i}\right\rangle+1} \in S(\mathbb{P})_{\omega_{0}}$ in $(10)$. Hence, it is easy to see that the sequences of homogeneous polynomials $F=\left(F_{0}, \ldots, F_{d}\right)$ and $F_{I}=\left(z_{i_{0}} \partial F_{0} / \partial z_{i_{0}}, \ldots, z_{i_{d}} \partial F_{0} / \partial z_{i_{d}}\right)$ are related by the formulae

$$
z_{i_{k}} \partial F_{0} / \partial z_{i_{k}}=F_{0}+\sum_{j=1}^{d}\left\langle m_{j}, e_{i_{k}}\right\rangle F_{j}=\sum_{j=0}^{d} A_{j k} F_{j}, \quad k=0, \ldots, d .
$$

Now, we have

$$
\begin{aligned}
\operatorname{Res}_{f}\left(t_{0}^{d} P\left(a_{1} t^{v_{1}}, \ldots, a_{n} t^{v_{n}}\right)\right) & =(-1)^{d-1} \operatorname{Res}_{F}\left(\tilde{q}(z) \cdot \prod_{i=1}^{r} z_{i}\right) \\
& =(-1)^{d-1} \operatorname{Res}_{F_{I}}\left(\operatorname{det}\left(A_{j k}\right) \tilde{q}(z) \cdot \prod_{i=1}^{r} z_{i}\right) \\
& =(-1)^{d-1} c_{I} \operatorname{Res}_{F_{I}}\left(\tilde{q}(z) \cdot \prod_{i=1}^{r} z_{i}\right)=(-1)^{d} Y_{Q}(a)
\end{aligned}
$$

where the second row follows from the Global Transformation Law for toric residue (see Theorem 9.8 below) and the third row follows from the equality $\operatorname{det}\left(A_{j k}\right)=$ $c_{I}$.

Next statement is a particular case of [CCD, Theorem 0.4].

Theorem 9.8. Let $G=\left(G_{0}, \ldots, G_{d}\right)$ and $H=\left(H_{0}, \ldots, H_{d}\right)$ be the regular sequences of elements in $S(\mathbb{P})_{\omega_{0}}$. If

$$
H_{j}=\sum_{i=0}^{d} A_{i j} G_{i}
$$

where $A_{i j}$ are complex numbers, then for each $P \in S(\mathbb{P})_{d \omega_{0}}$, we have

$$
\operatorname{Res}_{G}(P)=\operatorname{Res}_{H}\left(P \operatorname{det}\left(A_{i j}\right)\right) .
$$

Toric Residue Mirror Conjecture (Conjecture 4.6) implies the following:

Corollary 9.9. Let $\Delta \subset M_{\mathbb{R}}$ be an arbitrary reflexive d-dimensional polytope and $A$ a finite subset in $\Delta \cap M$ containing 0 and all vertices of $\Delta$. Choose any coherent triangulation $\mathcal{T}=\left\{\tau_{1}, \ldots, \tau_{k}\right\}$ of $\Delta$ associated with $A$ such that 0 is a vertex of all the simplices $\tau_{1}, \ldots, \tau_{k}$. Denote by $\mathbb{P}=\mathbb{P}_{\Sigma(\mathcal{T})}$ the simplicial toric variety defined 
by the fan $\Sigma=\Sigma(\mathcal{T}) \subset M_{\mathbb{R}}$ whose d-dimensional cones are exactly $\sigma_{i}:=\mathbb{R}_{\geq 0} \tau_{i}$ $(1 \leq i \leq k)$. If $A=\left\{0, v_{1}, \ldots, v_{n}\right\}$ and

$$
f(t):=1-\sum_{i=1}^{n} a_{i} t^{v_{i}}
$$

then for any homogeneous polynomial $Q\left(x_{1}, \ldots, x_{n}\right) \in \mathbb{Q}\left[x_{1}, \ldots, x_{n}\right]$ of degree $d-1$ the Laurent expansion of the $Q$-Yukawa $(d-1)$-point function $Y_{Q}(a)$ at the vertex $v_{\mathcal{T}} \in \operatorname{Sec}(A)$ corresponding to the coherent triangulations $\mathcal{T}$ coincides with the generating function of intersection numbers

$$
\sum_{\beta \in K_{\mathrm{eff}}(\mathbb{P})}\left\langle Q\left(\left[D_{1}\right], \ldots,\left[D_{n}\right]\right)\left(\left[D_{1}\right]+\cdots+\left[D_{n}\right]\right) \Phi_{\beta}\right\rangle_{\beta} a^{\beta},
$$

where the sum runs over all integral points $\beta=\left(b_{1}, \ldots, b_{n}\right)$ of the Mori cone $K_{\mathrm{eff}}(\mathbb{P})$, and $a^{\beta}:=a_{1}^{b_{1}} \cdots a_{n}^{b_{n}}$.

\section{Algorithmic CAlCulation of toric Residues}

An effective procedure for computing of toric residues in homogeneous coordinates using Gröbner basis calculus was developed in [CCD, CD]. Next we describe how these ideas can be used in concrete calculations of Yukawa $(d-1)$-point functions. We hold the same notations as in Section 9.

Definition 10.1 ( Cox1 ). Pick a subset $I=\left\{i_{1}, \ldots, i_{d}\right\} \subset\{1, \ldots, n\}$ such that $e_{i_{1}}, \ldots, e_{i_{d}}$ are linearly independent. Then define a toric Jacobian for any $(d+1)$ homogeneous polynomials $G_{0}, \ldots, G_{d} \in S(\mathbb{P})_{\alpha}$ by the formula

$$
J_{G}=\left(\begin{array}{ccc}
G_{0} & \cdots & G_{d} \\
\partial G_{0} / \partial z_{i_{1}} & \cdots & \partial G_{d} / \partial z_{i_{d}} \\
\vdots & \ddots & \vdots \\
\partial G_{0} / \partial z_{i_{d}} & \cdots & \partial G_{d} / \partial z_{i_{d}}
\end{array}\right) / \operatorname{det}\left(e_{I}\right) \widehat{z}_{I},
$$

where $e_{I}=\operatorname{det}\left(\left\langle m_{j}, e_{i_{k}}\right\rangle_{1 \leq j, k \leq d}\right)$ and $\widehat{z}_{I}=\prod_{i \notin I} z_{i}$.

Remark 10.2. Suppose that $G=\left(G_{0}, \ldots, G_{d}\right)$ is the image in $S(\mathbb{P})_{\alpha}$ of the regular sequence of elements in $S_{\Delta_{H}}^{1}$. Then it is easy to show (see [CDS) that the Jacobian $J_{G}$ in Definition 10.1 can be identified with the image of the Jacobian $J_{G}$ in (1) under the isomorphism (10).

As we have seen in the proof of Theorem 9.7, the function $Y_{Q}(a)$ is equal to the Cox's residue $-\operatorname{Res}_{F}\left(\tilde{q}(z) \cdot \prod_{i=1}^{r} z_{i}\right)$. We can compute this residue using the following method. Choose a Gröbner basis of the ideal generated by $F_{0}(z), \ldots, F_{d}(z) \in S(\mathbb{P})_{\omega_{0}}$. Then compute the normal form normalf $(H)$ for the polynomial $H=-\tilde{q}(z) \cdot \prod_{i=1}^{r} z_{i}$ and the normal form normalf $\left(J_{F}\right)$ for the toric 
Jacobian $J_{F}$. Since the quotient in (11) is one-dimensional, both normal forms are the multiples

$$
\operatorname{normalf}(H)=c z^{\sigma}, \quad \operatorname{normalf}\left(J_{F}\right)=c_{F} z^{\sigma}
$$

of some monomial $z^{\sigma} \in S(\mathbb{P})_{d \omega_{0}}$ by constants $c$ and $c_{F} \neq 0$ modulo $\left\langle F_{0}, \ldots, F_{d}\right\rangle$. Recall that the toric residue $\operatorname{Res}_{F}\left(J_{F}\right)$ is equal to the normalized volume $\operatorname{Vol}(\Delta)$ of polytope $\Delta$. Now given a polynomial $f(t)$ supported in the reflexive polytope $\Delta$ and a homogeneous polynomial $Q$, we get in output

$$
Y_{Q}(a)=\frac{c}{c_{F}} \operatorname{Vol}(\Delta)
$$

Example 10.3. We illustrate this method by the following example (cf. [COFKM, Section 8.1], [MP, Section 4.4], [HKTY, Appendix A.1], [CK, Example 5.6.2.1]). Let $\mathbb{P}(1,1,2,2,2)$ be the weighted projective space defined by the fan $\Sigma^{\prime} \subset M_{\mathbb{R}} \cong \mathbb{R}^{4}$ with one-dimensional generators

$$
\begin{aligned}
& v_{1}=(-1,-2,-2,-2), \quad v_{2}=(1,0,0,0), \quad v_{3}=(0,1,0,0), \\
& v_{4}=(0,0,1,0), \quad v_{5}=(0,0,0,1),
\end{aligned}
$$

which are the vertices of the reflexive polytope $\Delta \subset M_{\mathbb{R}}$. The polytope $\Delta$ contains only two lattice points except of listed above: $v_{6}=(0,-1,-1,-1)=\frac{1}{2}\left(v_{1}+v_{2}\right)$ and the origin $v_{0}=(0,0,0,0)$. Including the additional lattice point $v_{6}$ to the generators of $\Sigma^{\prime}$ corresponds to the blowup of $\mathbb{P}(1,1,2,2,2)$. The dual polytope $\Delta^{*} \subset N_{\mathbb{R}}$ to $\Delta$ is spanned by the vectors

$$
\begin{array}{ll}
e_{1}=(-1,-1,-1,-1), & e_{2}=(7,-1,-1,-1), \quad e_{3}=(-1,3,-1,-1), \\
e_{4}=(-1,-1,3,-1), & e_{5}=(-1,-1,-1,3),
\end{array}
$$

generating the fan $\Sigma \subset N_{\mathbb{R}}$ of $\mathbb{P}=\mathbb{P}_{\Sigma}$.

Take the Laurent polynomial

$$
f(t)=1-\sum_{i=1}^{6} a_{i} t^{v_{i}}=1-a_{1} t_{1}^{-1} t_{2}^{-2} t_{3}^{-2} t_{4}^{-2}-a_{2} t_{1}-a_{3} t_{2}-a_{4} t_{3}-a_{5} t_{4}-a_{6} t_{2}^{-1} t_{3}^{-1} t_{4}^{-1}
$$

having $\Delta$ as support polytope. After choosing the new variables

$$
y_{1}:=a_{3} a_{4} a_{5} a_{6}, \quad y_{2}:=\frac{a_{1} a_{2}}{a_{6}^{2}},
$$

we can put the equation for $f(t)$ in the form

$$
f(t)=1-y_{2} t_{1}^{-1} t_{2}^{-2} t_{3}^{-2} t_{4}^{-2}-t_{1}-y_{1} t_{2}-t_{3}-t_{4}-t_{2}^{-1} t_{3}^{-1} t_{4}^{-1} .
$$

Let $Z_{f}$ be a hypersurface in $\mathbb{T} \cong\left(\mathbb{C}^{*}\right)^{4}$ defined by $f(t)$ and forming the mirror family of Calabi-Yau hypersurfaces in $\mathbb{P}$. Denote

$$
\Omega:=\operatorname{Res}\left(\frac{1}{f} \frac{d t_{1}}{t_{1}} \wedge \cdots \wedge \frac{d t_{4}}{t_{4}}\right) \in H^{3}\left(Z_{f}\right) .
$$


We compute the Yukawa couplings

$$
Y^{(3-k, k)}\left(y_{1}, y_{2}\right)=\frac{-1}{(2 \pi i)^{3}} \int_{Z_{f}} \Omega \wedge\left(y_{1} \partial / \partial y_{1}\right)^{3-k}\left(y_{2} \partial / \partial y_{2}\right)^{k} \Omega, \quad k=0,1,2,3
$$

corresponding to polynomials $Q\left(x_{1}, x_{2}\right)=x_{1}^{3-k} x_{2}^{k}$. Show how to compute, say, the Yukawa coupling $Y^{(3,0)}\left(y_{1}, y_{2}\right)$ corresponding to the polynomial $Q\left(x_{1}, x_{2}\right)=x_{1}^{3}$. Let $z_{i}$ be the homogeneous coordinates on $\mathbb{P}$ related with the vectors $e_{i}(1 \leq i \leq 5)$. Then the homogenization of $f(t)$ defining the anticanonical hypersurface on $\mathbb{P}$ is

$$
F(z)=z_{1} z_{2} z_{3} z_{4} z_{5}-y_{2} z_{1}^{8}-z_{2}^{8}-y_{1} z_{3}^{4}-z_{4}^{4}-z_{5}^{4}-z_{1}^{4} z_{2}^{4} .
$$

Denote by $F_{1}(z), \ldots, F_{4}(z)$ the images of $t_{0} t_{1} \partial f / \partial t_{1}, \ldots, t_{0} t_{4} \partial f / \partial t_{4}$ under the isomorphism (10). Fix a Gröbner basis of the ideal $\left\langle F_{0}, \ldots, F_{4}\right\rangle$ with respect to the reverse lex order. Next, note that the homogenization of

$$
q(t)=-t_{0}^{3} Q\left(y_{1} t^{v_{1}}, y_{2} t^{v_{3}}\right)=-t_{0}^{3} Q\left(y_{1} t_{2}, y_{2} t_{1}^{-1} t_{2}^{-2} t_{3}^{-2} t_{4}^{-2}\right)=-t_{0}^{3}\left(y_{1} t_{2}\right)^{3}
$$

is $\tilde{q}(z)=-y_{1}^{3} z_{3}^{12}$. Applying the Gröbner basis calculation, we have found (using MAPLE) the following normal forms:

$$
\begin{aligned}
& \operatorname{normalf}\left(-\tilde{q}(z) \cdot z_{1} \cdots z_{5}\right)=4 y_{1}^{4} z_{3}^{16} \\
& \operatorname{normalf}\left(J_{F}\right)=4 y_{1}^{4}\left(\left(1-2^{8} y_{1}\right)^{2}-2^{18} y_{1}^{2} y_{2}\right) z_{3}^{16},
\end{aligned}
$$

where $J_{F}$ is the toric Jacobian. This easily yields that the Yukawa coupling is given by the formula

$$
Y^{(3,0)}\left(y_{1}, y_{2}\right)=\frac{8}{\left(1-2^{8} y_{1}\right)^{2}-2^{18} y_{1}^{2} y_{2}},
$$

since $\operatorname{Vol}(\Delta)=8$. Using the same procedure, we obtain

$$
\begin{aligned}
Y^{(2,1)}\left(y_{1}, y_{2}\right) & =\frac{4\left(1-2^{8} y_{1}\right)}{\left(1-2^{8} y_{1}\right)^{2}-2^{18} y_{1}^{2} y_{2}}, \\
Y^{(1,2)}\left(y_{1}, y_{2}\right) & =\frac{8 y_{2}\left(-1+2^{9} y_{1}\right)}{\left(1-4 y_{2}\right)\left(\left(1-2^{8} y_{1}\right)^{2}-2^{18} y_{1}^{2} y_{2}\right)}, \\
Y^{(0,3)}\left(y_{1}, y_{2}\right) & =\frac{4 y_{2}\left(1-2^{8} y_{1}+2^{2} y_{2}-2^{10} 3 y_{1} y_{2}\right)}{\left(1-4 y_{2}\right)^{2}\left(\left(1-2^{8} y_{1}\right)^{2}-2^{18} y_{1}^{2} y_{2}\right)} .
\end{aligned}
$$

The series expansions for $Y^{(3-k, k)}\left(y_{1}, y_{2}\right)$ were computed in [MP, Section 4.4]. The result of these computations is

$$
Y^{(3-k, k)}\left(y_{1}, y_{2}\right)=\sum_{\lambda_{1}, \lambda_{2} \geq 0} 2^{8 \lambda_{1}+2 \lambda_{2}+3-k}\left(\begin{array}{c}
\lambda_{1}+1-k \\
2 \lambda_{2}+1-k
\end{array}\right) y_{1}^{\lambda_{1}} y_{2}^{\lambda_{2}}, \quad k=0,1,2,3 .
$$


Now suppose that we do not blowup $\mathbb{P}(1,1,2,2,2)$. It means that we do not add $v_{6}$ to the generators of $\Sigma^{\prime}$. Then the polynomial

$$
f(t)=1-\sum_{i=1}^{5} a_{i} t^{v_{i}}=1-a_{1} t_{1}^{-1} t_{2}^{-2} t_{3}^{-2} t_{4}^{-2}-a_{2} t_{1}-a_{3} t_{2}-a_{4} t_{3}-a_{5} t_{4}
$$

can be transformed to

$$
f(t)=1-t_{1}^{-1} t_{2}^{-2} t_{3}^{-2} t_{4}^{-2}-y t_{1}-t_{2}-t_{3}-t_{4}
$$

after introducing the new variable $y:=a_{1} a_{2} a_{3}^{2} a_{4}^{2} a_{5}^{2}$. It is nice to observe that the Yukawa coupling

$$
Y^{3}(y)=\frac{8}{1-2^{18} y}=8+2097152 y+549755813888 y^{2}+O\left(y^{3}\right)
$$

for $Q(x)=x^{3}$ can be computed either by theorems 7.2 and 9.7 or as a limit $a_{6} \rightarrow 0$ in $Y^{(3,0)}\left(y_{1}, y_{2}\right)$ found above.

\section{Mirrors of Calabi-Yau hypersurfaces in $\mathbb{P}^{d_{1}} \times \mathbb{P}^{d_{2}}$}

Let us illustrate our method for computation of Yukawa $(d-1)$-point functions for Calabi-Yau hypersurfaces $Z_{f} \subset \mathbb{T} \cong\left(\mathbb{C}^{*}\right)^{2}$ defined by polynomial

$$
f(t)=1-\sum_{i_{1}=1}^{n_{1}} a_{1 i_{1}} t^{v_{1 i_{1}}}-\sum_{i_{2}=1}^{n_{2}} a_{2 i_{2}} t^{v_{2 i_{2}}},
$$

where the exponents $v_{i j}$ satisfy the relations

$$
v_{11}+\cdots+v_{1 n_{1}}=0, \quad v_{21}+\cdots+v_{2 n_{2}}=0 .
$$

The toric variety $\mathbb{P}_{\Sigma}$ with the fan $\Sigma$ of dimension $d=d_{1}+d_{2}$ (here, $d_{1}=n_{1}-1, d_{2}=$ $\left.n_{2}-1\right)$ with generators $\left\{v_{j n_{j}}\right\}$ is the product of two projective spaces $\mathbb{P}^{d_{1}} \times \mathbb{P}^{d_{2}}$. According to Bat2], the hypersurfaces $Z_{f}$ form the mirror family of Calabi-Yau hypersurfaces in $\mathbb{P}^{d_{1}} \times \mathbb{P}^{d_{2}}$.

Denote by $y_{1}=a_{11} \cdots a_{1 n_{1}}, y_{2}=a_{21} \cdots a_{2 n_{2}}$ the new variables and by $\theta_{1}=$ $y_{1} \partial / \partial y_{1}, \theta_{2}=y_{2} \partial / \partial y_{2}$ the logarithmic partial derivations. Given a form-residue

$$
\Omega:=\operatorname{Res}\left(\frac{1}{f} \frac{d t_{1}}{t_{1}} \wedge \cdots \wedge \frac{d t_{d}}{t_{d}}\right) \in H^{d-1}\left(Z_{f}\right),
$$

the 2-parameter Yukawa $(d-1)$-point functions are defined as the integrals

$$
Y^{\left(k_{1}, k_{2}\right)}\left(y_{1}, y_{2}\right)=\frac{(-1)^{\frac{(d-1)(d-2)}{2}}}{(2 \pi i)^{d-1}} \int_{Z_{f}} \Omega \wedge \theta_{1}^{k_{1}} \theta_{2}^{k_{2}} \Omega, \quad k_{1}+k_{2}=d-1 .
$$

Theorem 9.7, Theorem 8.1 and Proposition 8.3 easily implies the following statement. 
Proposition 11.1. The Yukawa $(d-1)$-point function $Y^{\left(k_{1}, k_{2}\right)}\left(y_{1}, y_{2}\right)$ is equal to the toric residue related with the polynomial $P\left(x_{1}, x_{2}\right)=x_{1}^{k_{1}} x_{2}^{k_{2}}\left(n_{1} x_{1}+n_{2} x_{2}\right)$. Let $u_{1}=n_{1}^{n_{1}} y_{1}, u_{2}=n_{2}^{n_{2}} y_{2}$. Then the function $Y^{\left(k_{1}, k_{2}\right)}\left(y_{1}, y_{2}\right)$ can be computed

1) as a trace of rational function:

$$
Y^{\left(k_{1}, k_{2}\right)}\left(u_{1}, u_{2}\right)=n_{1}^{d_{1}-k_{1}-1} n_{2}^{d_{2}-k_{2}-1} \sum_{\substack{z_{1}^{n_{1}}=u_{1} \\ z_{2}^{n_{2}}=u_{2}}} \frac{1}{z_{1}^{d_{1}-k_{1}} z_{2}^{d_{2}-k_{2}}\left(1-z_{1}-z_{2}\right)}
$$

2) as a series:

$$
Y^{\left(k_{1}, k_{2}\right)}\left(u_{1}, u_{2}\right)=n_{1}^{d_{1}-k_{1}} n_{2}^{d_{2}-k_{2}} \sum_{b_{1}, b_{2} \geq 0} \frac{\left(n_{1} b_{1}+n_{2} b_{2}+1\right) !}{\left(n_{1} b_{1}+d_{1}-k_{1}\right) !\left(n_{2} b_{2}+d_{2}-k_{2}\right) !} u_{1}^{b_{1}} u_{2}^{b_{2}} .
$$

Some examples of explicit calculation of Yukawa $(d-1)$-point functions as rational functions are given below.

Example 11.2. $\mathbb{P}^{1} \times \mathbb{P}^{1} ; u_{1}=2^{2} y_{1}, u_{2}=2^{2} y_{2}$.

$$
\begin{aligned}
Y^{(1,0)}\left(u_{1}, u_{2}\right) & =\frac{1}{2} \sum_{\substack{z_{1}^{2}=u_{1} \\
z_{2}^{2}=u_{2}}} \frac{1}{z_{2}\left(1-z_{1}-z_{2}\right)}=2 \sum_{b_{1}, b_{2} \geq 0} \frac{\left(2 b_{1}+2 b_{2}+1\right) !}{\left(2 b_{1}\right) !\left(2 b_{2}+1\right) !} u_{1}^{b_{1}} u_{2}^{b_{2}} \\
& =\frac{2\left(1+u_{1}-u_{2}\right)}{\left(1-u_{1}-u_{2}\right)^{2}-4 u_{1} u_{2}} .
\end{aligned}
$$

By symmetry, $Y^{(0,1)}\left(u_{1}, u_{2}\right)=Y^{(1,0)}\left(u_{2}, u_{1}\right)$.

Example 11.3. $\mathbb{P}^{1} \times \mathbb{P}^{2} ; u_{1}=2^{2} y_{1}, u_{2}=3^{3} y_{2}$.

$$
\begin{aligned}
Y^{(2,0)}\left(u_{1}, u_{2}\right) & =\frac{3}{2} \sum_{\substack{z_{1}^{2}=u_{1} \\
z_{2}^{3}=u_{2}}} \frac{z_{1}}{z_{2}^{2}\left(1-z_{1}-z_{2}\right)}=\frac{9}{2} \sum_{b_{1}, b_{2} \geq 0} \frac{\left(2 b_{1}+3 b_{2}+1\right) !}{\left(2 b_{1}-1\right) !\left(3 b_{2}+2\right) !} u_{1}^{b_{1}} u_{2}^{b_{2}} \\
& =\frac{9}{2} \frac{u_{1}\left(3+u_{1}\right)}{\left(1-u_{1}\right)^{3}-2 u_{2}\left(1-3 u_{1}\right)} . \\
Y^{(1,1)}\left(u_{1}, u_{2}\right) & =\frac{1}{2} \sum_{z_{1}^{2}=u_{1}} \frac{1}{z_{2}\left(1-z_{1}-z_{2}\right)}=3 \sum_{b_{1}, b_{2} \geq 0} \frac{\left(2 b_{1}+3 b_{2}+1\right) !}{\left(2 b_{1}\right) !\left(3 b_{2}+1\right) !} u_{1}^{b_{1}} u_{2}^{b_{2}} \\
& =\frac{3\left(1-u_{2}-u_{1}^{2}\right)}{\left(1-u_{1}\right)^{3}-2 u_{2}\left(1-3 u_{1}\right)} .
\end{aligned}
$$




$$
\begin{aligned}
Y^{(0,2)}\left(u_{1}, u_{2}\right) & =\frac{1}{3} \sum_{\substack{z_{1}^{2}=u_{1} \\
z_{2}^{3}=u_{2}}} \frac{1}{z_{1}\left(1-z_{1}-z_{2}\right)}=2 \sum_{b_{1}, b_{2} \geq 0} \frac{\left(2 b_{1}+3 b_{2}+1\right) !}{\left(2 b_{1}+1\right) !\left(3 b_{2}\right) !} u_{1}^{b_{1}} u_{2}^{b_{2}} \\
& =\frac{2\left(\left(1-u_{1}\right)^{2}+2 u_{2}\right)}{\left(1-u_{1}\right)^{3}-2 u_{2}\left(1-3 u_{1}\right)} .
\end{aligned}
$$

The next example of hypersurfaces in product of projective spaces was considered in [BvS] in the case of diagonal one-parameter subfamily and in [HKTY].

Example 11.4. $\mathbb{P}^{2} \times \mathbb{P}^{2} ; u_{1}=3^{3} y_{1}, u_{2}=3^{3} y_{2}$.

$$
\begin{aligned}
Y^{(3,0)}\left(u_{1}, u_{2}\right) & =\frac{1}{3} \sum_{\substack{z_{1}^{3}=u_{1} \\
z_{2}^{3}=u_{2}}} \frac{z_{1}}{z_{2}^{2}\left(1-z_{1}-z_{2}\right)}=3 \sum_{b_{1}, b_{2} \geq 0} \frac{\left(3 b_{1}+3 b_{2}+1\right) !}{\left(3 b_{1}-1\right) !\left(3 b_{2}+2\right) !} u_{1}^{b_{1}} u_{2}^{b_{2}} \\
& =\frac{9 u_{1}\left(2+u_{1}+u_{2}\right)}{\left(1-u_{1}-u_{2}\right)^{3}-27 u_{1} u_{2}} . \\
Y^{(2,1)}\left(u_{1}, u_{2}\right)= & \frac{1}{3} \sum_{\substack{z_{1}^{3}=u_{1} \\
z_{2}^{3}=u_{2}}} \frac{1}{z_{2}\left(1-z_{1}-z_{2}\right)}=3 \sum_{b_{1}, b_{2} \geq 0} \frac{\left(3 b_{1}+3 b_{2}+1\right) !}{\left(3 b_{1}\right) !\left(3 b_{2}+1\right) !} u_{1}^{b_{1}} u_{2}^{b_{2}} \\
= & \frac{3\left(\left(1-u_{2}\right)^{2}+u_{1}\left(1-2 u_{1}-u_{2}\right)\right)}{\left(1-u_{1}-u_{2}\right)^{3}-27 u_{1} u_{2}} .
\end{aligned}
$$

By symmetry, $Y^{\left(k_{1}, k_{2}\right)}\left(u_{1}, u_{2}\right)=Y^{\left(k_{2}, k_{1}\right)}\left(u_{2}, u_{1}\right)$.

\section{REFERENCES}

[Bat1] V.V. Batyrev, Variations of the mixed Hodge structure of affine hypersurfaces in algebraic tori, Duke Math. J. 69 (1993), 349-409.

[Bat2] _ Dual polyhedra and mirror symmetry for Calabi-Yau hypersurfaces in toric varieties, J. Algebraic Geom. 3 (1994), 493-535.

[Bat3] Stringy Hodge numbers of varieties with Gorenstein canonical singularities. Integrable systems and algebraic geometry (Kobe/Kyoto, 1997), 1-32, World Sci. Publishing, River Edge, NJ, 1998.

[BC] V.V. Batyrev, D. Cox, On the Hodge structure of projective hypersurfaces in toric varieties, Duke Math. J. 75 (1995), 293-338.

[BvS] V.V. Batyrev, D. van Straten, Generalized hypergeometric functions and rational curves on Calabi-Yau complete intersections in toric varieties, Commun. Math. Phys. 168 (1995), 493-533.

[BM] V.V. Batyrev, E.N. Materov, Mixed toric residues and Calabi-Yau complete intersections, in preparation.

[COFKM] P. Candelas, X. de la Ossa, A. Font, S. Katz, D.R. Morrison, Mirror symmetry for two parameter model (I), Nuclear Phys. B 416 (1994), 481-562. 
[COGP] P. Candelas, X. de la Ossa, P. Green, L. Parkes, A pair of Calabi-Yau manifolds as an exactly solvable superconformal theory, Nuclear Phys. B 359 (1991), 24-74.

[CD] E. Cattani, A. Dickenstein, A global view of residues in the torus, J. of Pure and Appl. Algebra 117 \& 118 (1997), 119-144.

[CCD] E. Cattani, D. Cox and A. Dickenstein, Residues in toric varieties, Compositio Math. 108 (1997), 35-76.

[CDS] E. Cattani, A. Dickenstein and B. Sturmfels, Residues and resultants, J. Math. Sci. Univ. Tokyo 5 (1998), 119-148.

[Cox1] D. Cox, The homogeneous coordinate ring of a toric variety, J. Algebraic Geom. 4 (1995), 17-50.

[Cox2] Toric residues, Ark. Mat. 34 (1996), 73-96.

[CK] D. Cox, S. Katz Mirror Symmetry and Algebraic Geometry, Math. Surveys Monogr. 68, Amer. Math. Soc., Providence, 1999.

[CR1] W. Chen, Y. Ruan, Orbifold quantum cohomology. Preprint version available at http://arxiv.org/abs/math.AG/0005198.

[CR2] Orbifold Gromov-Witten theory. Preprint version available at http://arxiv.org/abs/math.AG/0103156.

[Dan] V.I. Danilov, The geometry of toric varieties, Russian Math. Surveys 33 (1978), 95154 .

[Di] A. Dickenstein, Hypergeometric functions with integer homogeneities, to appear: Proc. Meeting on Complex Analysis (June - July 1998), eds. F. Norguet, S. Ofman, International Press.

[GKZ] I.M. Gelfand, M.M. Kapranov and A.V. Zelevinsky, Discriminants, Resultants and Multidimensional Determinants, Birkhäuser, Boston, 1994.

[GKh] O.A. Gelfond, A.G. Khovanskii, Toric geometry and Grothendieck residues, Moscow Math. J. 2 (2002), no. 2, 101-114.

[G1] A. Givental, Homological geometry and mirror symmetry, Chatterji, S. D. (ed.), Proceedings of the international congress of mathematicians, ICM '94, August 3-11, 1994, Zrich, Switzerland. Vol. I. Basel: Birkhäuser. (1995), 472-480.

[G2] _ Homological geometry I: Projective hypersurfaces, Sel. Math., New Ser. 1, no. 2, (1995), 325-345.

[G3] , Equivariant Gromov-Witten invariants, Int. Math. Res. Not. 1996, no. 13, (1996), 613-663.

[G4] A mirror theorem for toric complete intersections, Kashiwara, Masaki (ed.) et al., Topological field theory, primitive forms and related topics. Proceedings of the 38th Taniguchi symposium, Kyoto, Japan, December 9-13, 1996 and the RIMS symposium with the same title, Kyoto, Japan, December 16-19, 1996. Boston, MA: Birkhuser. Prog. Math. 160, (1998), 141-175.

[G5] - The mirror formula for quintic threefolds, Eliashberg, Ya. (ed.) et al., Northern California symplectic geometry seminar. Providence, RI: American Mathematical Society. Transl., Ser. 2, Am. Math. Soc. 196(45) (1999), 49-62.

[Ha1] R. Hartshorne, Algebraic Geometry, Springer-Verlag, 1977.

[Ha2] , Residues and Duality, Lecture Notes in Math. 20, Springer-Verlag, Berlin Heidelberg New York, 1966.

[HKTY] S. Hosono, A. Klemm, S. Theisen and S.-T. Yau, Mirror symmetry, Mirror map and applications to Calabi-Yau hypersurfaces, Commun. Math. Phys. 167 (1995), 301-350. 
[JN] M. Jinzenji, M. Nagura, Mirror Symmetry and an exact calculation of $N-2$ point correlation function on Calabi-Yau manifold embedded in $\mathbb{C P}^{N-1}$, Int. J. Mod. Phys. A11 (1996), 1217-1252.

[LLY1] B. Lian, K. Liu, S.-T. Yau, Mirror principle I, Asian J. Math. 1 (1997), no. 4, 729-763.

[LLY2] — Mirror principle II, Asian J. Math. 3 (1999), no. 1, 109-146.

[LLY3] - Mirror principle III, Asian J. Math. 3 (1999), no. 4, 771-800.

[Mav1] A.R. Mavlyutov, Semiample hypersurfaces in toric varieties, Duke Math. J. 101 (2000), 85-116.

[Mav2] - On the chiral ring of Calabi-Yau hypersurfaces in toric varieties. Preprint version available at http://arxiv.org/abs/preprint/math.AG/0010318.

[MP] D. Morrison, M. Plesser, Summing the instantons: Quantum cohomology and mirror symmetry in toric varieties, Nucl. Phys. B 440 (1995), 279-354.

Mathematisches Institut, Universität Tübingen, Auf der Morgenstelle 10, TüBINGEn D-72076, GeRmany

E-mail address: victor.batyrev@uni-tuebingen.de

Mathematisches Institut, Universität Tübingen, Auf Der Morgenstelle 10, TüBIngen D-72076, Germany

E-mail address: evgeny.materov@uni-tuebingen.de 